Network Working Group

Request for Comments: 3635

Obsoletes: 2665

Category: Standards Track
J. Flick

Hewlett-Packard Company

September 2003

\title{
Definitions of Managed Objects for \\ the Ethernet-like Interface Types
}

Status of this Memo

This document specifies an Internet standards track protocol for the Internet community, and requests discussion and suggestions for improvements. Please refer to the current edition of the "Internet Official Protocol Standards" (STD 1) for the standardization state and status of this protocol. Distribution of this memo is unlimited.

Copyright Notice

Copyright (C) The Internet Society (2003). All Rights Reserved.

Abstract

This memo defines a portion of the Management Information Base (MIB) for use with network management protocols in the Internet community. In particular, it defines objects for managing Ethernet-like interfaces. This memo obsoletes RFC 2665. It updates that specification by including management information useful for the management of 10 Gigabit per second (Gb/s) Ethernet interfaces.

Table of Contents

1. Introduction . . . . . . . . . . . . . . . . . . . . . . . 2

2. The Internet-Standard Management Framework . . . . . . . . . 3

3. Overview . . . . . . . . . . . . . . . . . . . . . 3

3.1. Relation to MIB-2. . . . . . . . . . . . . . . . . . . . 4

3.2. Relation to the Interfaces MIB . . . . . . . . . . . . . 4

3.2.1. Layering Model . . . . . . . . . . . . . . 4

3.2.2. Virtual Circuits. . . . . . . . . . . . 4

3.2.3. ifRcvAddresstable. . . . . . . . . . . . 5

3.2.4. iftype . . . . . . . . . . . . . . . . 5

3.2.5. ifXxxOctets. . . . . . . . . . . . 5

3.2.6. ifXxxXcastPkts . . . . . . . . . 6

3.2.7. ifMtu. . . . . . . . . . . . . . . . 8

3.2.8. ifspeed and ifHighspeed. . . . . . . . . . . 8

3.2.9. ifphysAddress. . . . . . . . . . . . . . . . . 9

3.2.10. Specific Interface MIB Objects. . . . . . . . 10

3.3. Relation to the 802.3 MAU MIB. . . . . . . . . . . . 13 
3.4. dot3statsEtherChipset. . . . . . . . . . . . . . . 13

3.5. Mapping of IEEE 802.3 Managed Objects. . . . . . . . 14

4. Definitions. . . . . . . . . . . . . . . . . . . . . . . . . 17

5. Intellectual Property Statement. . . . . . . . . . . . . . 55

6. Acknowledgements . . . . . . . . . . . . . . . . . . 56

7. Normative References . . . . . . . . . . . . . . . . 57

8. Informative References . . . . . . . . . . . . . . . 58

9. Security Considerations. . . . . . . . . . . . . . . . . 59

10. IANA Considerations. • . . . . . . . . . . . . . . . . . . . 60

A. Change Log . . . . . . . . . . . . . . . . . . . . . 61

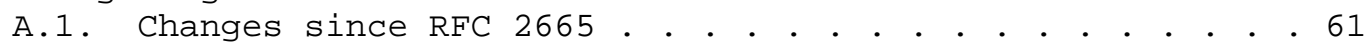

A.2. Changes between $\operatorname{RFC} 2358$ and $\operatorname{RFC} 2665$. . . . . . . . $\quad$. 62

A.3. Changes between RFC 1650 and RFC 2358. . . . . . . . . 62

Author's Address . . . . . . . . . . . . . . . . . . 63

Full Copyright Statement . . . . . . . . . . . . . . . . . 64

1. Introduction

This memo defines a portion of the Management Information Base (MIB) for use with network management protocols in the Internet community. In particular, it defines objects for managing Ethernet-like interfaces.

This memo also includes a MIB module. This MIB module updates the list of managed objects specified in the earlier version of this MIB, module, RFC 2665 [RFC2665].

Ethernet technology, as defined by the 802.3 Working Group of the IEEE, continues to evolve, with scalable increases in speed, new types of cabling and interfaces, and new features. This evolution may require changes in the managed objects in order to reflect this new functionality. This document, as with other documents issued by this working group, reflects a certain stage in the evolution of Ethernet technology. In the future, this document might be revised, or new documents might be issued by the Ethernet Interfaces and Hub MIB Working Group, in order to reflect the evolution of Ethernet technology

The key words "MUST", "MUST NOT", "REQUIRED", "SHALL", "SHALL NOT", "SHOULD", "SHOULD NOT", "RECOMMENDED", "MAY", and "OPTIONAL" in this document are to be interpreted as described in [RFC2119]. 


\section{The Internet-Standard Management Framework}

For a detailed overview of the documents that describe the current Internet-Standard Management Framework, please refer to section 7 of RFC 3410 [RFC3410].

Managed objects are accessed via a virtual information store, termed the Management Information Base or MIB. MIB objects are generally accessed through the Simple Network Management Protocol (SNMP). Objects in the MIB are defined using the mechanisms defined in the Structure of Management Information (SMI). This memo specifies a MIB module that is compliant to the SMIV2, which is described in STD 58, RFC 2578 [RFC2578], STD 58, RFC 2579 [RFC2579] and STD 58, RFC 2580 [RFC2580].

3. Overview

Instances of these object types represent attributes of an interface to an ethernet-like communications medium. At present, ethernet-like media are identified by the value ethernetcsmacd(6) of the iftype object in the Interfaces MIB [RFC2863]. Some older implementations may return the values iso88023Csmacd(7) or starLan(11) for iftype for ethernet-like media.

The definitions presented here are based on Section 30, "10 Mb/s, 100 $\mathrm{Mb} / \mathrm{s} 1000 \mathrm{Mb} / \mathrm{s}$ and $10 \mathrm{~Gb} / \mathrm{s}$ Management", and Annex 30A, "GDMO Specification for 802.3 managed object classes" of IEEE Std. 802.3, 2002 Edition [IEEE802.3], amended by IEEE Std. 802.3ae-2002 [IEEE802.3ae], as originally interpreted by Frank Kastenholz, then of Interlan in [KASTEN]. Implementors of these MIB objects should note that IEEE Std. 802.3 [IEEE802.3] explicitly describes (in the form of Pascal pseudocode) when, where, and how various MAC attributes are measured. The IEEE document also describes the effects of MAC actions that may be invoked by manipulating instances of the MIB objects defined here.

To the extent that some of the attributes defined in [IEEE802.3] are represented by previously defined objects in MIB-2 [RFC1213] or in the Interfaces MIB [RFC2863], such attributes are not redundantly represented by objects defined in this memo. Among the attributes represented by objects defined in other memos are the number of octets transmitted or received on a particular interface, the number of frames transmitted or received on a particular interface, the promiscuous status of an interface, the MAC address of an interface, and multicast information associated with an interface. 


\subsection{Relation to MIB-2}

This section applies only when this MIB is used in conjunction with the "old" [RFC1213] interface group.

The relationship between an ethernet-like interface and an interface in the context of MIB-2 is one-to-one. As such, the value of an ifIndex object instance can be directly used to identify corresponding instances of the objects defined herein.

For agents which implement the (now deprecated) ifspecific object, an instance of that object that is associated with an ethernet-like interface has the OBJECT IDENTIFIER value:

$$
\text { dot } 3 \text { OBJECT IDENTIFIER }:=\{\text { transmission } 7\}
$$

\subsection{Relation to the Interfaces MIB}

The Interface MIB [RFC2863] requires that any MIB which is an adjunct of the Interface MIB clarify specific areas within the Interface MIB. These areas were intentionally left vague in the Interface MIB to avoid over constraining the MIB, thereby precluding management of certain media-types.

Section 4 of [RFC2863] enumerates several areas which a media-specific MIB must clarify. Each of these areas is addressed in a following subsection. The implementor is referred to [RFC2863] in order to understand the general intent of these areas.

\subsubsection{Layering Model}

Ordinarily, there are no sublayers for an ethernet-like interface. However there may be implementation-specific requirements which require the use of sublayers. One example is the use of $802.3 \mathrm{link}$ aggregation. In this case, Annex 30C of [IEEE802.3] describes the layering model and the use of the ifstackTable for representing aggregated links. Another example is the use of the 802.3 WAN Interface Sublayer. In this case, The 802.3 WIS MIB [RFC3637] describes the layering model and the use of the ifstackTable for representing the WAN sublayer.

\subsubsection{Virtual Circuits}

This medium does not support virtual circuits and this area is not applicable to this MIB. 


\subsection{3. ifRcvAddressTable}

This table contains all IEEE 802.3 addresses, unicast, multicast, and broadcast, for which this interface will receive packets and forward them up to a higher layer entity for local consumption. The format of the address, contained in ifRcvAddressAddress, is the same as for ifPhysAddress.

In the event that the interface is part of a MAC bridge, this table does not include unicast addresses which are accepted for possible forwarding out some other port. This table is explicitly not intended to provide a bridge address filtering mechanism.

\subsection{4. iftype}

This MIB applies to interfaces which have the iftype value ethernetCsmacd(6). It is REQUIRED that all ethernet-like interfaces use an iftype of ethernetCsmacd(6) regardless of the speed that the interface is running or the link-layer encapsulation in use. Use of the iftype values iso88023Csmacd(7) and starLan(11) are deprecated, however some older implementations may return these values. Management applications should be prepared to receive these deprecated iftype values from older implementations.

There are three other interface types defined in the IANAifType-MIB for Ethernet. They are fastEther(62), fastEtherFX(69), and gigabitEthernet(117). These interface types were registered by individual vendors, not by any IETF working group. A requirement for compliance with this document is that all ethernet-like interfaces MUST return ethernetCsmacd(6) for ifType, and MUST NOT return fastEther(62), fastEtherFX(69), or gigabitEthernet(117). However, as there are fielded implementations that do return these obsolete ifType values, management applications SHoULD be prepared to receive them from older implementations.

Information on the particular flavor of Ethernet that an interface is running is available from ifspeed in the Interfaces MIB, and ifMauType in the 802.3 MAU MIB [RFC3636]. Note that implementation of the 802.3 MAU MIB [RFC3636] is REQUIRED for all ethernet-like interfaces.

\subsection{5. ifxxxOctets}

The Interface MIB octet counters, ifInoctets, ifoutoctets, ifHCInOctets and ifHCOutOctets, MUST include all octets in valid frames sent or received on the interface, including the MAC header and FCS, but not the preamble, start of frame delimiter, or extension octets. This corresponds to the definition of framesize/8 in section 
4.2.7.1 of [IEEE802.3] (frameSize is defined in bits rather than octets, and is defined as 2 x addressize + lengthorTypesize + datasize + crcSize). They do not include the number of octets in collided or failed transmit attempts, since the MAC layer driver typically does not have visibility to count these octets. They also do not include octets in received invalid frames, since this information is normally not passed to the MAC layer, and since non-promiscuous MAC implementations cannot reliably determine whether an invalid frame was actually addressed to this station.

Note that these counters do include octets in valid MAC control frames sent or received on the interface, as well as octets in otherwise valid received MAC frames that are discarded by the MAC layer for some reason (insufficient buffer space, unknown protocol, etc.).

Note that the octet counters in IF-MIB do not exactly match the definition of the octet counters in IEEE 802.3. aOctetsTransmittedOK and aOctetsReceivedoK count only the octets in the clientData and Pad fields, whereas ifInOctets and ifoutoctets include the entire MAC frame, including MAC header and FCS. However, the IF-MIB counters can be derived from the IEEE 802.3 counters as follows:

$$
\begin{aligned}
& \text { ifInOctets }=\text { aOctetsReceivedOK }+(18 * \text { aFramesReceivedOK }) \\
& \text { ifOutoctets }=\text { aOctetsTransmittedOK }+(18 * \text { aFramesTransmittedOK })
\end{aligned}
$$

Another difference to keep in mind between the IF-MIB counters and IEEE 802.3 counters is that in the IEEE 802.3 document, the frame counters and octet counters are always incremented together. aOctetsTransmittedoK counts the number of octets in frames that were counted by aFramesTransmittedoK. aOctetsReceivedOK counts the number of octets in frames that were counted by aFramesReceivedok. This is not the case with the IF-MIB counters. The IF-MIB octet counters count the number of octets sent to or received from the layer below this interface, whereas the packet counters count the number of packets sent to or received from the layer above. Therefore, received MAC Control frames, ifInDiscards, and ifInUnknownProtos are counted by ifInOctets, but not ifInXcastPkts. Transmitted MAC Control frames are counted by ifoutoctets, but not ifoutxcastPkts. ifOutDiscards and ifOutErrors are counted by ifoutXcastPkts, but not ifoutoctets.

\subsection{6. ifXxxXcastPkts}

The packet counters in the IF-MIB do not exactly match the definition of the frame counters in IEEE 802.3. aFramesTransmittedoK counts the number of frames successfully transmitted on the interface, whereas ifoutUcastPkts, ifoutMulticastPkts and ifoutBroadcastPkts count the 
number of transmit requests made from a higher layer, whether or not the transmit attempt was successful. This means that packets counted by ifOutErrors or ifoutDiscards are also counted by ifoutxcastPkts, but are not counted by aFramestransmittedoK. This also means that, since MAC Control frames are generated by a sublayer internal to the interface layer rather than by a higher layer, they are not counted by ifoutXcastPkts, but are counted by aFramesTransmittedok. Roughly:

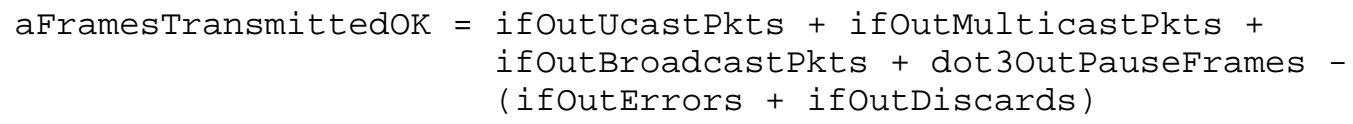

Similarly, aFramesReceivedoK counts the number of frames received successfully by the interface, whether or not they are passed to a higher layer, whereas ifInUcastPkts, ifInMulticastPkts and ifInBroadcastpkts count only the number of packets passed to a higher layer. This means that packets counted by ifInDiscards or ifInUnknownProtos are also counted by aFramesReceivedok, but are not counted by ifInXcastPkts. This also means that, since MAC Control frames are consumed by a sublayer internal to the interface layer and not passed to a higher layer, they are not counted by ifInXcastPkts, but are counted by aFramesReceivedoK. Roughly:

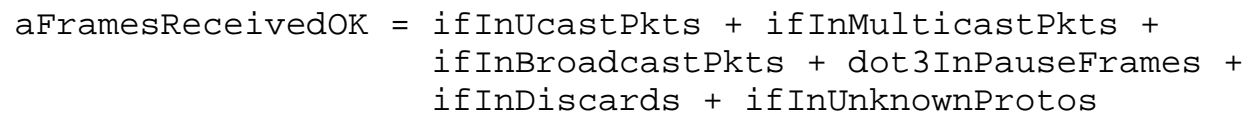

This specification chooses to treat MAC control frames as being originated and consumed within the interface and not counted by the IF-MIB packet counters. MAC control frames are normally sent as multicast packets. In many network environments, MAC control frames can greatly outnumber multicast frames carrying actual data. If MAC control frames were included in the ifInMulticastPkts and ifoutMulticastPkts, the count of data-carrying multicast packets would tend to be drowned out by the count of MAC control frames, rendering those counters considerably less useful.

To better understand the issues surrounding the mapping of the IF-MIB packet and octet counters to an Ethernet interface, it is useful to refer to a Case Diagram [CASE] for the IF-MIB counters, with modifications to show the proper interpretation for the Ethernet interface layer. 
layer above

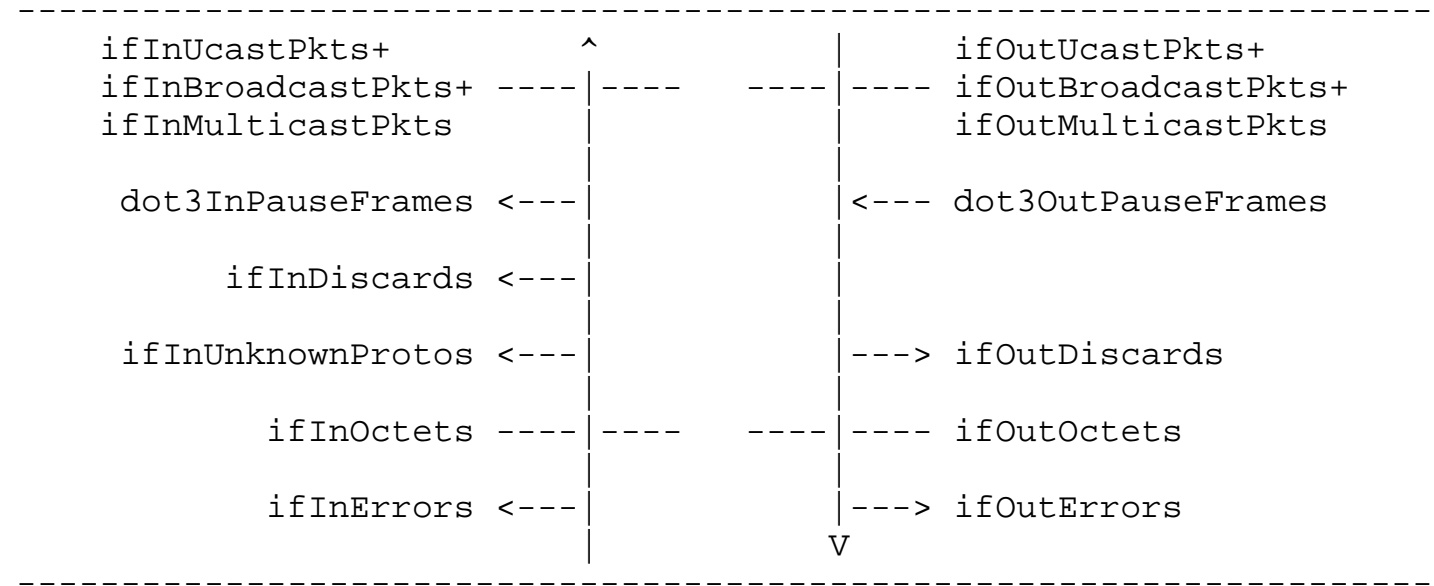

layer below

\subsection{7. ifmtu}

The defined standard MTU for ethernet-like interfaces is 1500 octets. However, many implementations today support larger packet sizes than the IEEE 802.3 standard. The value of this object MUST reflect the actual MTU in use on the interface, whether it matches the standard MTU or not.

This value should reflect the value seen by the MAC client interface. When a higher layer protocol, like IP, is running over Ethernet framing, this is the MTU that will be seen by that higher layer protocol. However, most ethernet-like interfaces today run multiple protocols that use a mix of different framing types. For example, an IEEE 802.2 LLC type 1 client protocol will see an MTU of 1497 octets on an interface using the IEEE standard maximum packet size, and a protocol running over SNAP will see an MTU of 1492 octets on an interface using the IEEE standard maximum packet size. However, since specification mandates using the MTU as seen at the MAC client interface, the value of ifMtu would be reported as 1500 octets in these cases.

\subsection{8. ifspeed and ifHighspeed}

For ethernet-like interfaces operating at 1000 Megabits per second $(\mathrm{Mb} / \mathrm{s})$ or less, ifspeed will represent the current operational speed of the interface in bits per second. For current interface types, this will be equal to 1,000,000 (1 million), 10,000,000 (10 million), $100,000,000$ (100 million), or 1,000,000,000 (1 billion). ifHighspeed will represent the current operational speed in millions of bits per 
second. For current ethernet-like interfaces, this will be equal to 1, 10, 100, or 1,000. If the interface implements auto-negotiation, auto-negotiation is enabled for this interface, and the interface has not yet negotiated to an operational speed, these objects SHOULD reflect the maximum speed supported by the interface.

For ethernet-like interfaces operating at greater than $1000 \mathrm{Mb} / \mathrm{s}$, ifHighspeed will represent the current operational speed of the interface in millions of bits per second. Note that for WAN implementations, this will be the payload data rate over the WAN interface sublayer. For current implementations, this will be equal to 10,000 for LAN implementations of $10 \mathrm{~Gb} / \mathrm{s}$, and 9,294 for WAN implementations of the $10 \mathrm{~Gb} / \mathrm{s}$ MAC over an OC-192 PHY. For these speeds, ifspeed should report a maximum unsigned 32-bit value of $4,294,967,295$ as specified in [RFC2863].

Note that these object MUST NOT indicate a doubled value when operating in full-duplex mode. It MUST indicate the correct line speed regardless of the current duplex mode. The duplex mode of the interface may be determined by examining either the dot3statsDuplexstatus object in this MIB module, or the ifMauType object in the 802.3 MAU MIB [RFC3636].

\subsection{9. ifPhysAddress}

This object contains the IEEE 802.3 address which is placed in the source-address field of any Ethernet, Starlan, or IEEE 802.3 frames that originate at this interface. Usually this will be kept in ROM on the interface hardware. Some systems may set this address via software.

In a system where there are several such addresses the designer has a tougher choice. The address chosen should be the one most likely to be of use to network management (e.g. the address placed in ARP responses for systems which are primarily IP systems).

If the designer truly can not chose, use of the factory-provided ROM address is suggested.

If the address can not be determined, an octet string of zero length should be returned.

The address is stored in binary in this object. The address is stored in "canonical" bit order, that is, the Group Bit is positioned as the low-order bit of the first octet. Thus, the first byte of a multicast address would have the bit 0x01 set. 


\subsubsection{Specific Interface MIB Objects}

The following table provides specific implementation guidelines for applying the interface group objects to ethernet-like media.

object

ifIndex

ifDescr

iftype

ifMtu

ifspeed

ifPhysAddress

ifAdminstatus

ifoperstatus

ifLastChange

ifInoctets

ifInUcastPkts
Guidelines

Each ethernet-like interface is represented by an ifEntry. The dot3statsTable in this MIB module is indexed by dot3statsIndex. The interface identified by a particular value of dot3statsIndex is the same interface as identified by the same value of ifIndex.

Refer to [RFC2863].

Refer to section 3.2.4.

Refer to section 3.2.7.

Refer to section 3.2.8.

Refer to section 3.2.9.

Write access is not required. Support for 'testing' is not required.

The operational state of the interface. Support for 'testing' is not required. The value 'dormant' has no meaning for an ethernet-like interface.

Refer to [RFC2863].

The number of octets in valid MAC frames received on this interface, including the MAC header and FCS. This does include the number of octets in valid MAC Control frames received on this interface. See section 3.2.5.

Refer to [RFC2863]. Note that this does not include MAC Control frames, since MAC Control frames are consumed by the interface layer and are not passed to any higher layer protocol. See section 3.2 .6 . 
ifInDiscards

ifInErrors

ifInUnknownProtos

ifoutoctets

ifOutUcastPkts

ifoutDiscards

ifoutErrors

ifName

ifInMulticastPkts

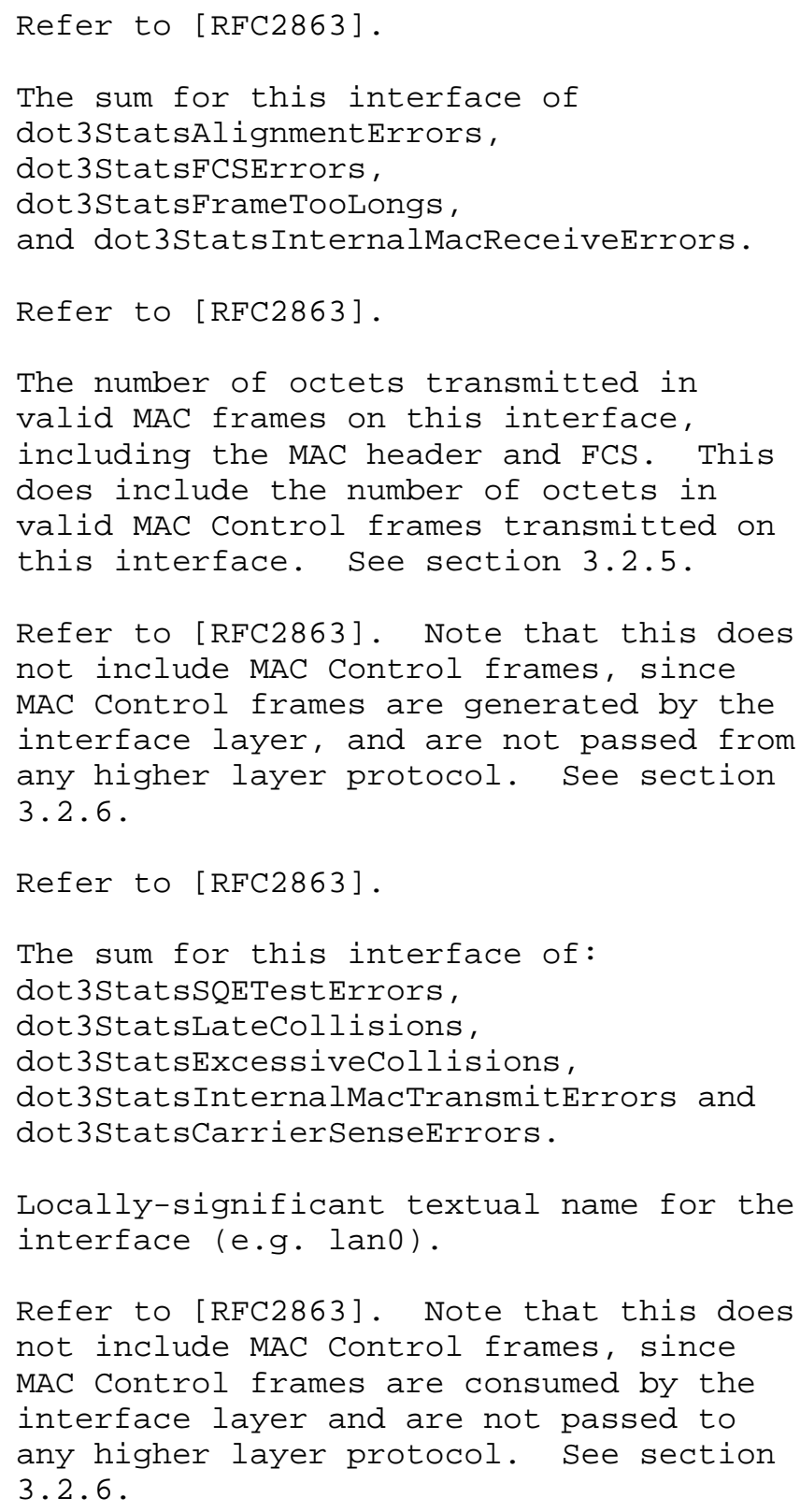


ifInBroadcastPkts

ifOutMulticastPkts

ifOutBroadcastPkts

ifHCInOctets

ifHCOutoctets
ifAlias

ifHCInUcastPkts ifHCInMulticastPkts ifHCInBroadcastPkts ifHCOutUcastPkts ifHCOutMulticastPkts ifHCOutBroadcastPkts

ifLinkUpDownTrapEnable

ifHighspeed

ifPromiscuousMode

ifConnectorPresent

Flick
Refer to [RFC2863]. Note that this does not include MAC Control frames, since MAC Control frames are consumed by the interface layer, and are not passed to any higher layer protocol. See section 3.2 .6 .

Refer to [RFC2863]. Note that this does not include MAC Control frames, since MAC Control frames are generated by the interface layer, and are not passed from any higher layer protocol. See section 3.2 .6 .

Refer to [RFC2863]. Note that this does not include MAC Control frames, since MAC Control frames are generated by the interface layer, and are not passed from any higher layer protocol. See section 3.2 .6 .

64-bit versions of counters. Required for ethernet-like interfaces that are capable of operating at $20 \mathrm{Mb} / \mathrm{s}$ or faster, even if the interface is currently operating at less than $20 \mathrm{Mb} / \mathrm{s}$.

64-bit versions of packet counters. Required for ethernet-like interfaces that are capable of operating at $640 \mathrm{Mb} / \mathrm{s}$ or faster, even if the interface is currently operating at less than $640 \mathrm{Mb} / \mathrm{s}$.

Refer to [RFC2863]. Default is ' enabled'

Refer to section 3.2.8.

Refer to [RFC2863].

This will normally be 'true'. It will be 'false' in the case where this interface uses the WAN Interface Sublayer. See [RFC3637] for details.

Refer to [RFC2863]. 
ifCounterDiscontinuityTime Refer to [RFC2863]. Note that a discontinuity in the Interface MIB counters may also indicate a discontinuity in some or all of the counters in this MIB that are associated with that interface.

ifstackHigherLayer Refer to section 3.2.1. ifStackLowerLayer ifstackstatus

ifRcvAddressAddress

Refer to section 3.2.3. ifRcvAddressitatus ifRcvAddress Type

\subsection{Relation to the 802.3 MAU MIB}

Support for the mauModIfCompl3 compliance statement of the MAU-MIB [RFC3636] is REQUIRED for Ethernet-like interfaces. This MIB is needed in order to allow applications to determine the current MAU type in use by the interface, and to control autonegotiation and duplex mode for the interface. Implementing this MIB module without implementing the MAU-MIB would leave applications with no standard way to determine the media type in use, and no standard way to control the duplex mode of the interface.

\section{4. dot3statsEtherChipset}

This document defines an object called dot3statsEtherchipset, which is used to identify the MAC hardware used to communicate on an interface. Previous versions of this document contained a number of OID assignments for some existing Ethernet chipsets. Maintaining that list as part of this document has proven to be problematic, so the OID assignments contained in previous versions of this document have now been moved to a separate document [RFC2666].

The dot3StatsEtherChipset object has now been deprecated. Implementation feedback indicates that this object is much more useful in theory than in practice. The object's utility in debugging network problems in the field appears to be limited. In those cases where it may be useful, it is not sufficient, since it identifies only the MAC chip, and not the PHY, PMD, or driver. The administrative overhead involved in maintaining a central registry of chipset OIDs cannot be justified for an object whose usefulness is questionable at best. 
Implementations which continue to support this object for the purpose of backwards compatibility may continue to use the values defined in [RFC2666]. For chipsets not listed in [RFC2666], implementors that wish to support this object and return a valid OBJECT IDENTIFIER value may assign OBJECT IDENTIFIERS within that part of the registration tree delegated to individual enterprises.

\subsection{Mapping of IEEE 802.3 Managed Objects}

IEEE 802.3 Managed Object

oMacEntity

- aMACID

- aFramesTransmittedoK

- aSinglecollisionframes

- aMultiplecollisionframes

. aFramesReceivedoK

- aFrameChecksequenceErrors

- aAlignmentErrors

- aOctetsTransmittedoK

- aFramesWithDeferredXmissions

-aLatecollisions

- aFramesAbortedDueToXsColls

- aFramesLostDueToIntMACXmitError

- aCarrierSenseErrors

. aOctetsReceivedoK

- aFramesLostDueToIntMACRcvError

- aPromiscuousstatus

- aReadMulticastAddressList

- aMulticastFramesXmittedOK

- aBroadcastFramesXmittedOK

- aMulticastFramesReceivedOK

- aBroadcastFramesReceivedOK

- aFrameTooLongErrors

- aReadWriteMACAddress

- aCollisionframes

- aDuplexstatus

- aRateControlAbility

- aRateControlstatus

- acAddGroupAddress

- acDeleteGroupAddress

-acExecuteSelfTest
Corresponding SNMP Object

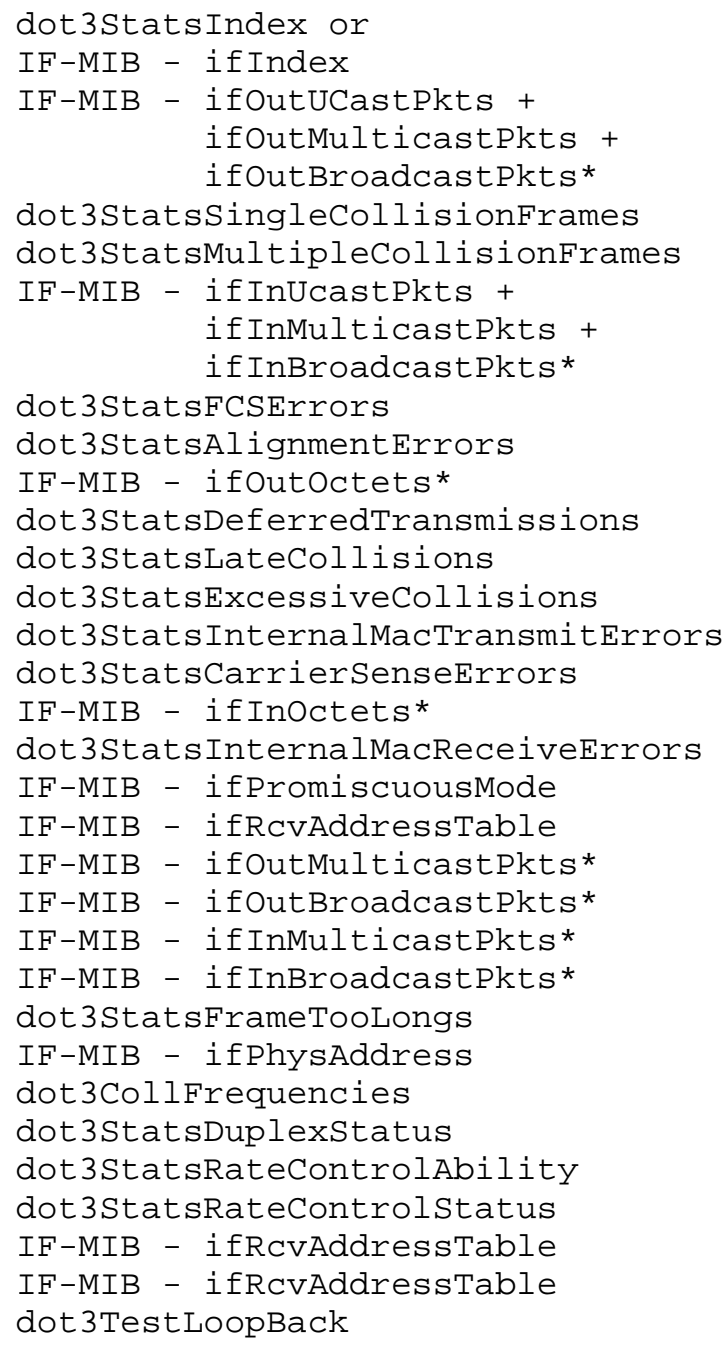




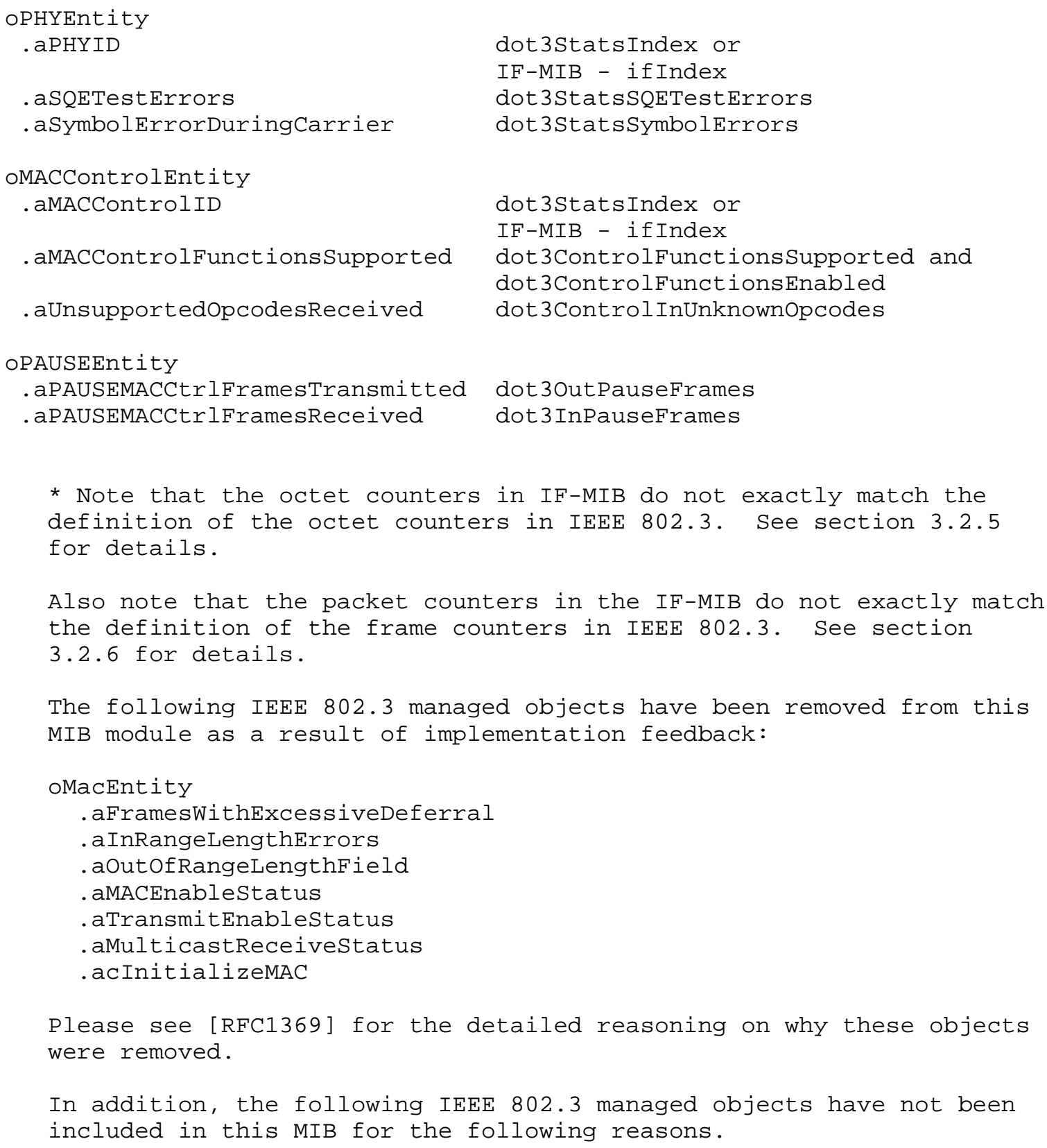


IEEE 802.3 Managed Object

oMACEntity - aMACCapabilities

- astretchRatio

oPHYEntity -aphytype

-aPhyTypeList

-aMIIDetect

.aPhyAdminstate

- acPhyAdmincontrol

oMACControlentity

- aMACControlFramesTransmitted

. aMACControlframesReceived

oPAUSEEntity - aPAUSELinkDelayAl lowance
Disposition

Can be derived from

MAU-MIB - ifMauTypeListBits

Implementation constant.

Can be derived from

MAU-MIB - ifMauType

Can be derived from

MAU-MIB - ifMauTypeListBits

Not considered useful.

Can already obtain interface state from IF-MIB - ifAdminstatus and MAU state from MAU-MIB ifMaustatus. Providing an additional state for the PHY was not considered useful.

Can already control interface state from IF-MIB - ifAdminstatus and MAU state from MAU-MIB ifMaustatus. Providing separate admin control of the PHY was not considered useful.

Can be determined by summing the OutFrames counters for the individual control functions

Can be determined by summing the Inframes counters for the individual control functions

Not considered useful. 
4. Definitions

EtherLike-MIB DEFINITIONS ::= BEGIN

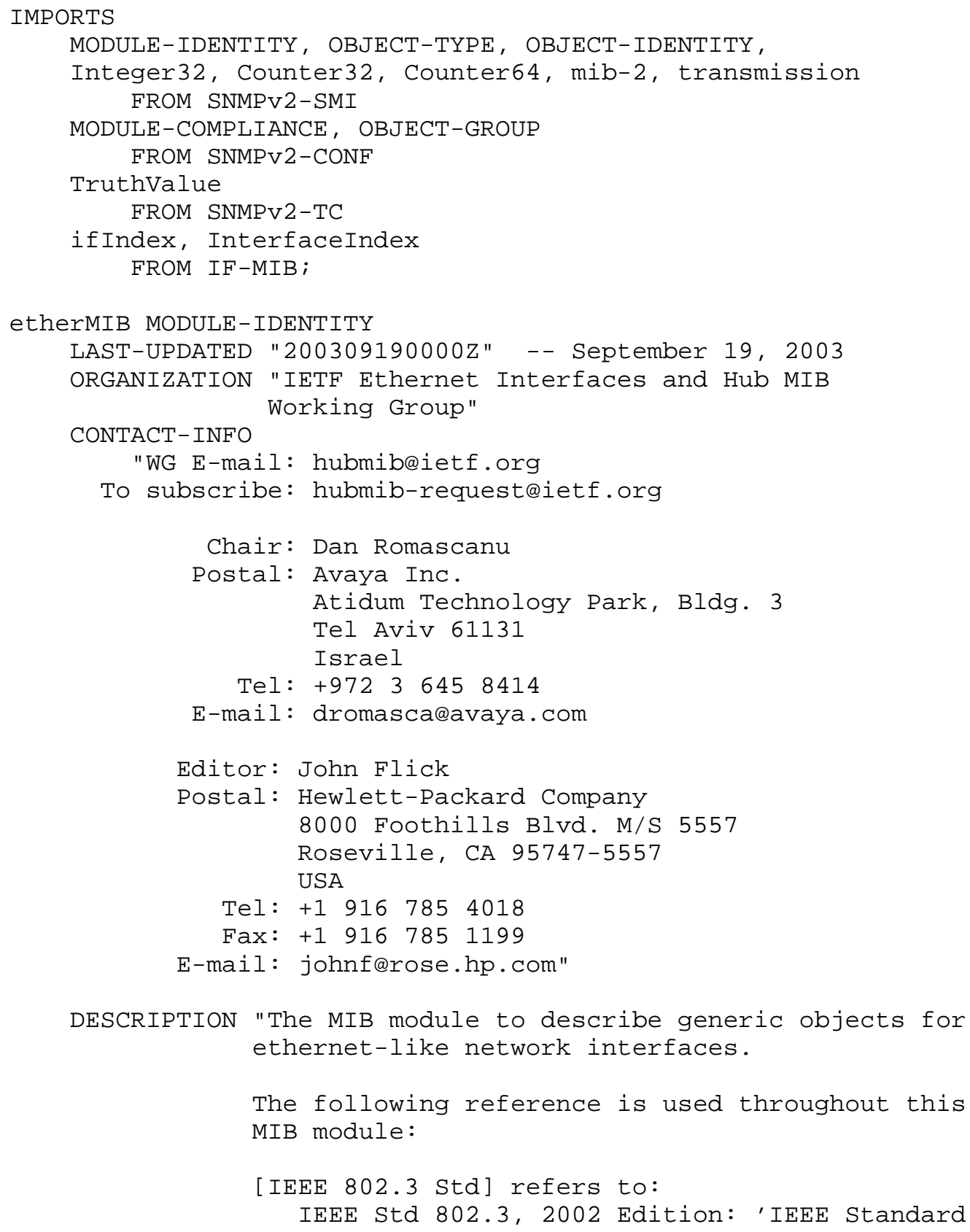

Flick 


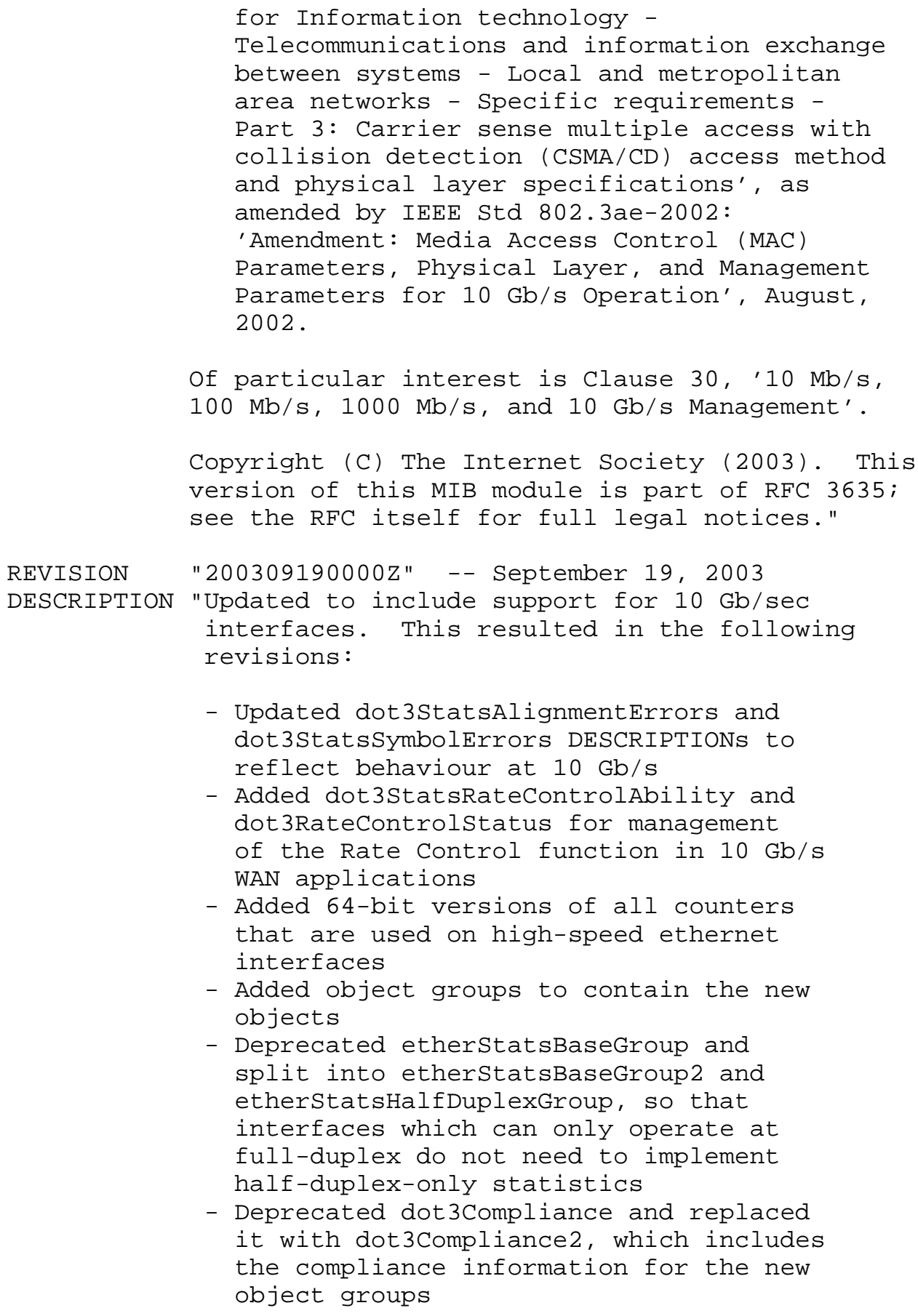

- Updated dot3statsAlignmentErrors and dot3statsSymbolerrors DESCRIPTIONs to reflect behaviour at $10 \mathrm{~Gb} / \mathrm{s}$

- Added dot3statsRateControlAbility and dot3RateControlstatus for management of the Rate Control function in $10 \mathrm{~Gb} / \mathrm{s}$ WAN applications

- Added 64-bit versions of all counters that are used on high-speed ethernet interfaces

- Added object groups to contain the new objects

- Deprecated etherStatsBaseGroup and split into etherStatsBaseGroup2 and etherStatsHalfDuplexGroup, so that interfaces which can only operate at full-duplex do not need to implement half-duplex-only statistics

- Deprecated dot3Compliance and replaced it with dot3compliance2, which includes the compliance information for the new object groups 


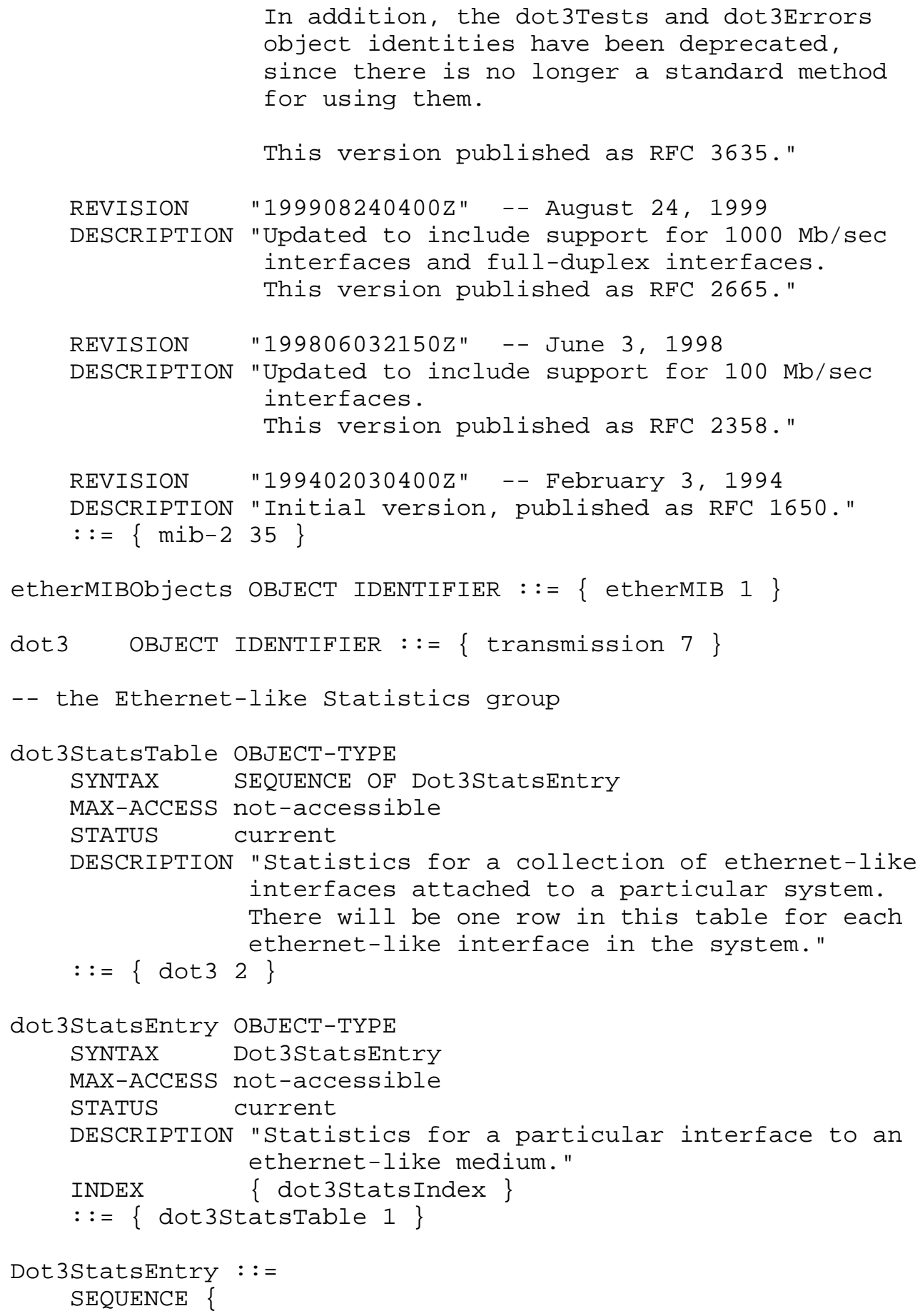

Flick 


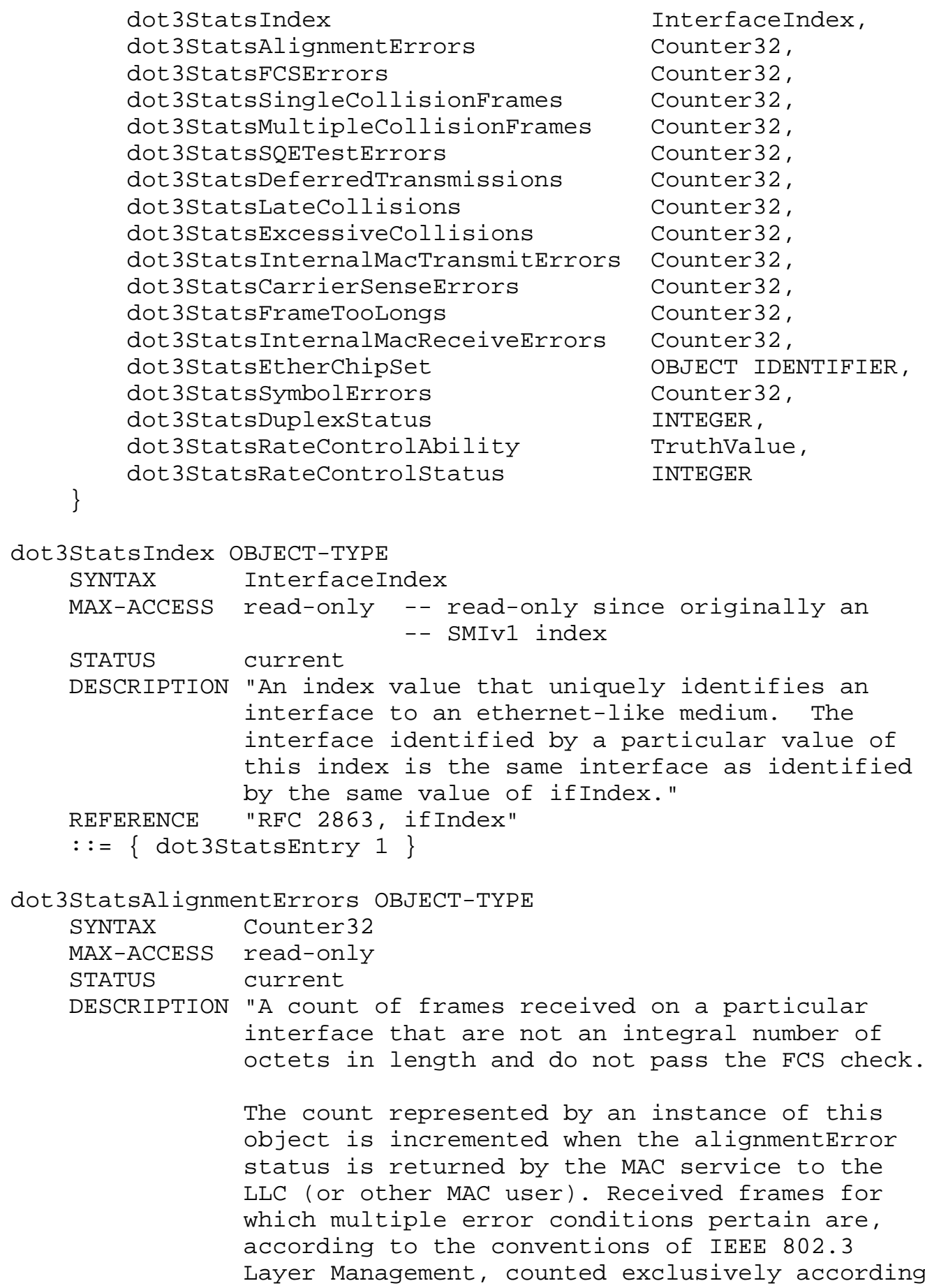




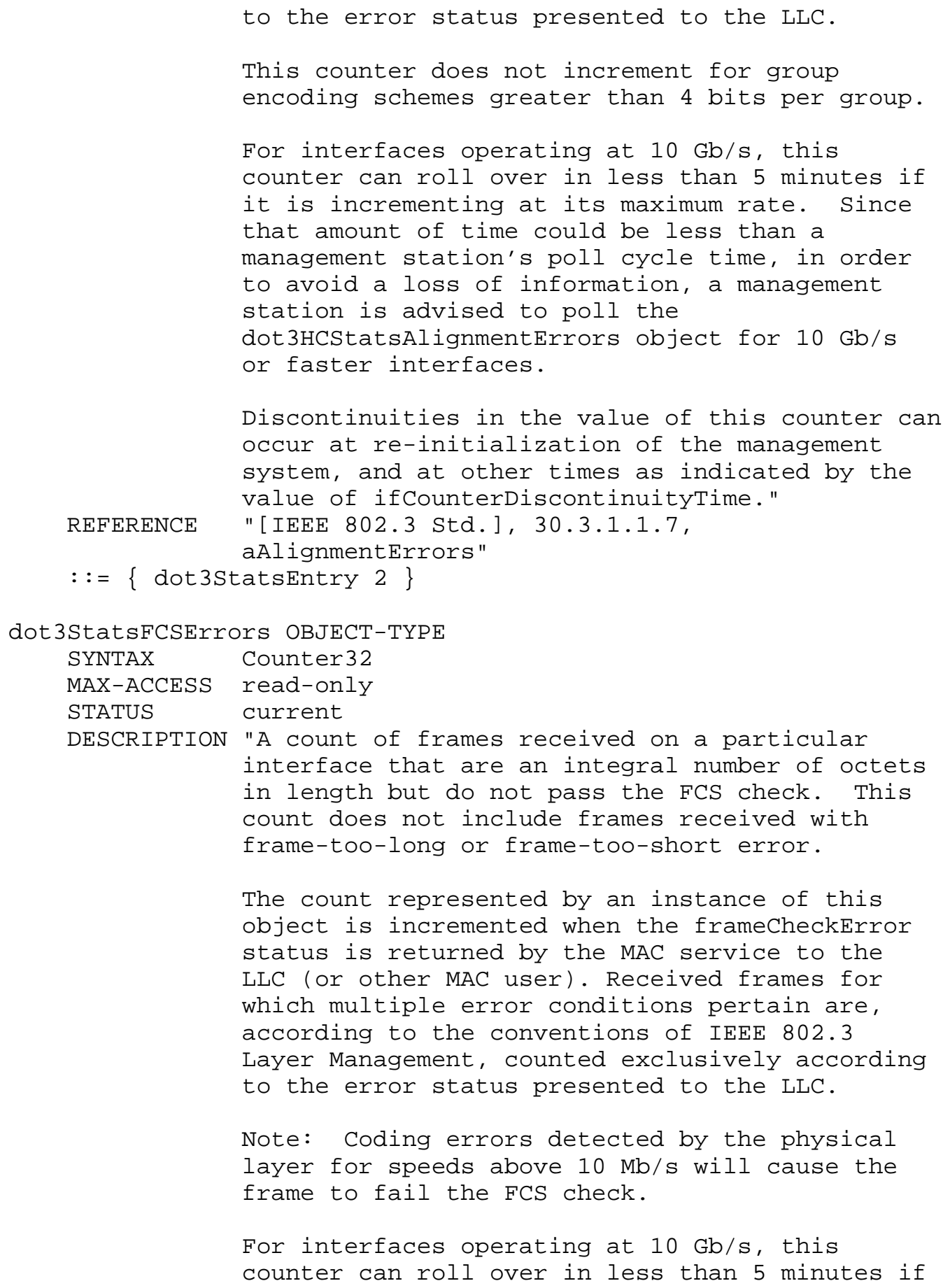




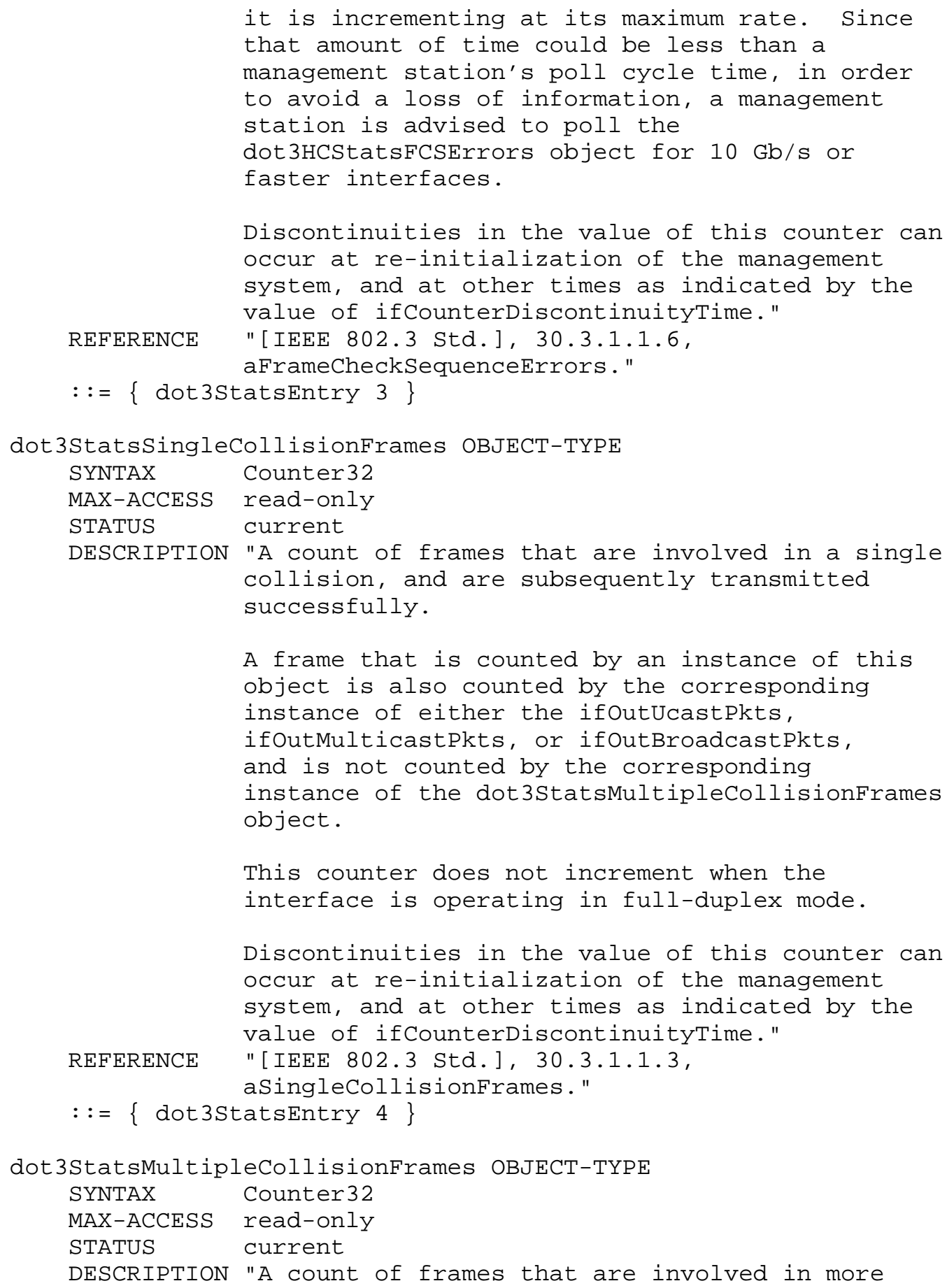




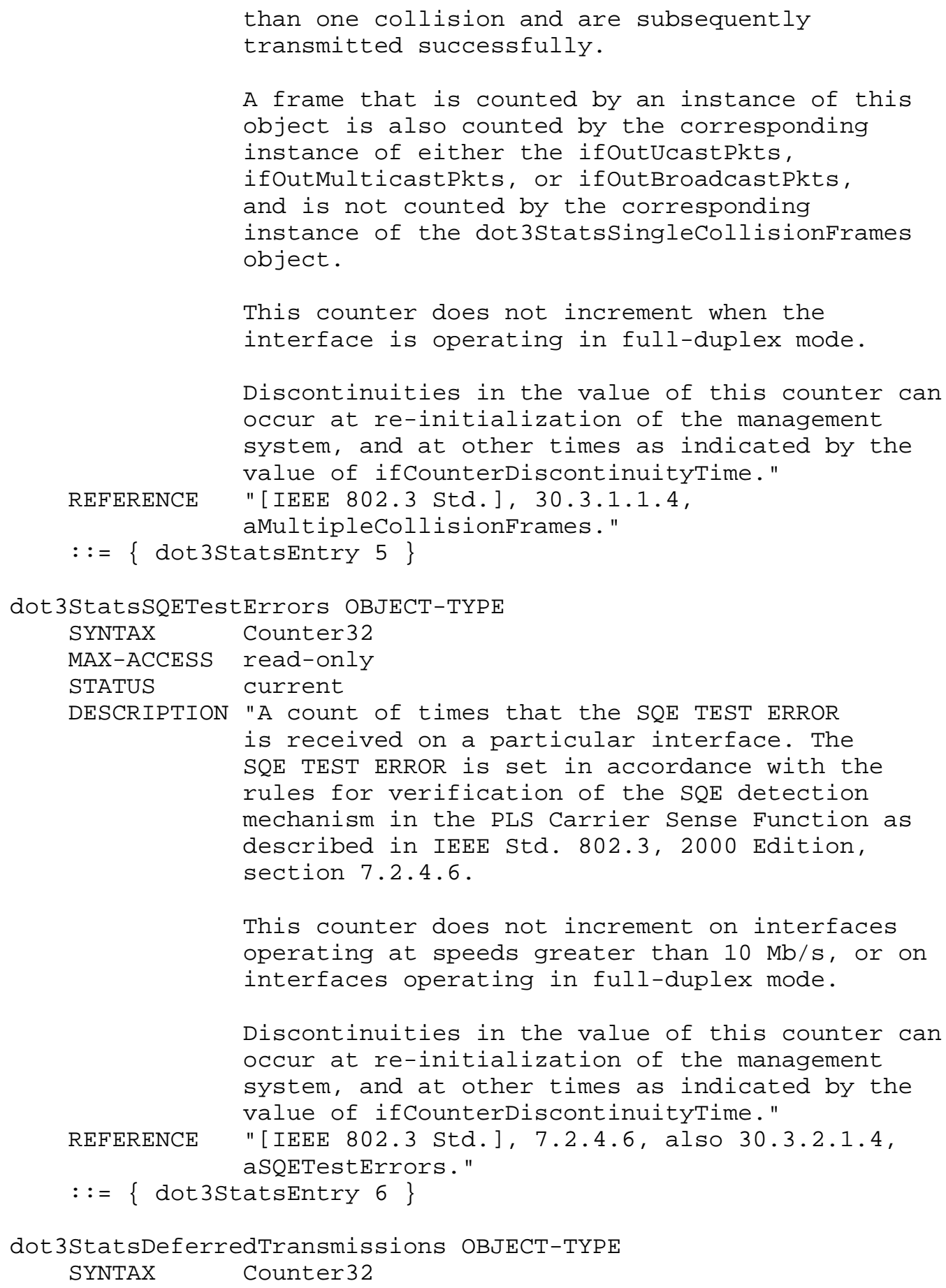




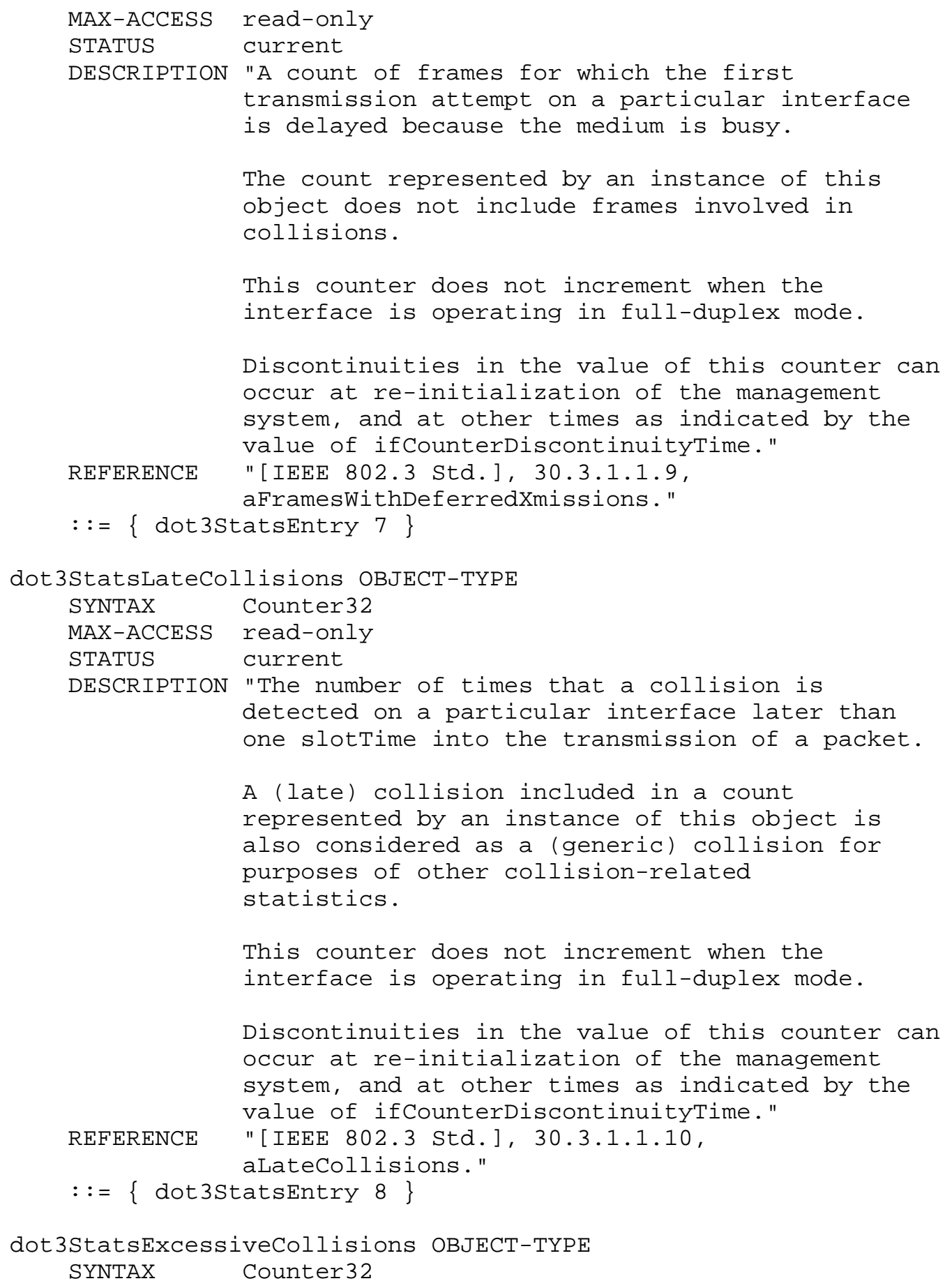




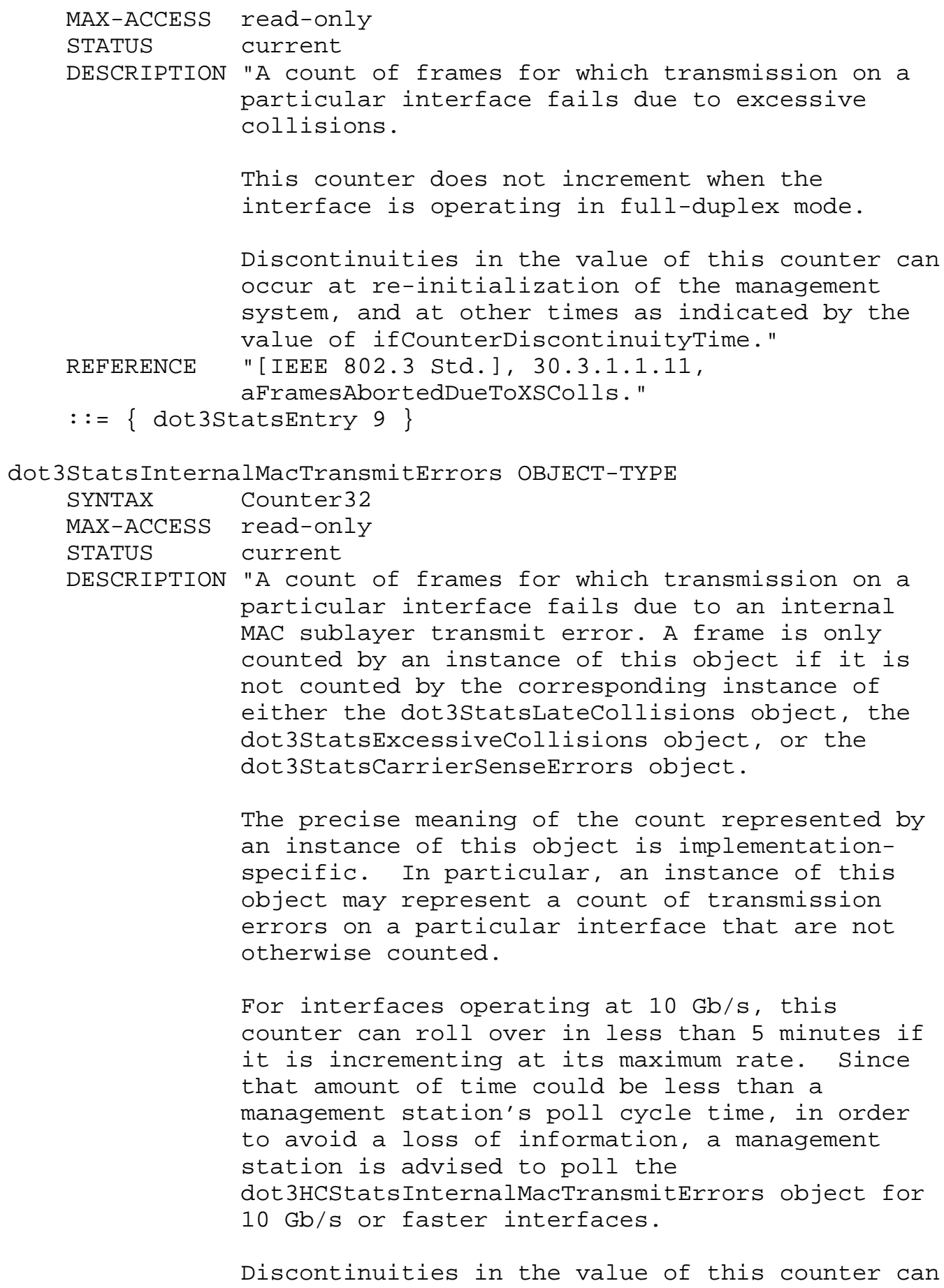




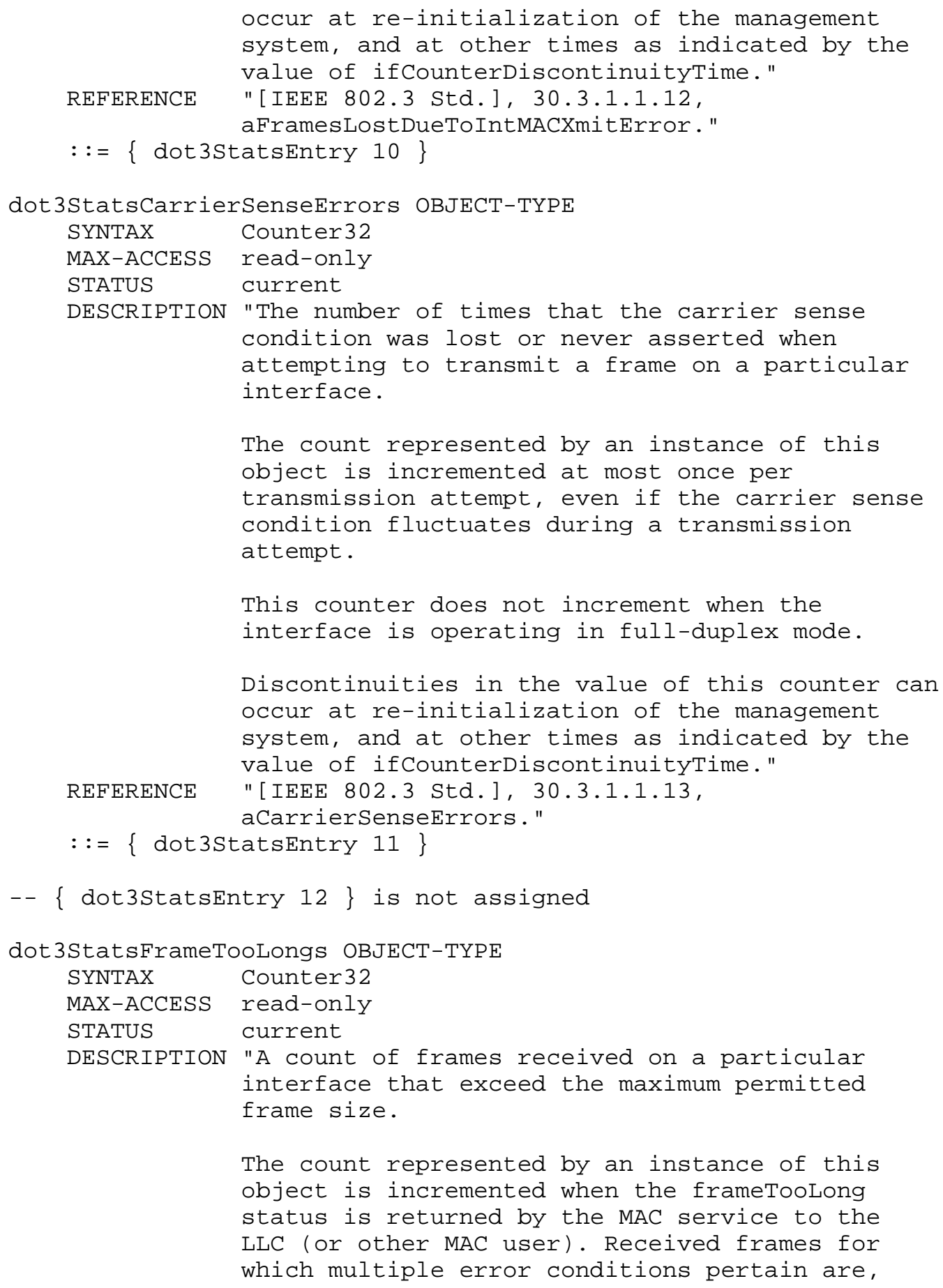

Flick 


\author{
according to the conventions of IEEE 802.3 \\ Layer Management, counted exclusively according \\ to the error status presented to the LLC. \\ For interfaces operating at $10 \mathrm{~Gb} / \mathrm{s}$, this \\ counter can roll over in less than 80 minutes if \\ it is incrementing at its maximum rate. Since \\ that amount of time could be less than a \\ management station's poll cycle time, in order \\ to avoid a loss of information, a management \\ station is advised to poll the \\ dot3HCStatsFrameTooLongs object for $10 \mathrm{~Gb} / \mathrm{s}$ \\ or faster interfaces. \\ Discontinuities in the value of this counter can \\ occur at re-initialization of the management \\ system, and at other times as indicated by the \\ value of ifCounterDiscontinuityTime."

Flick 


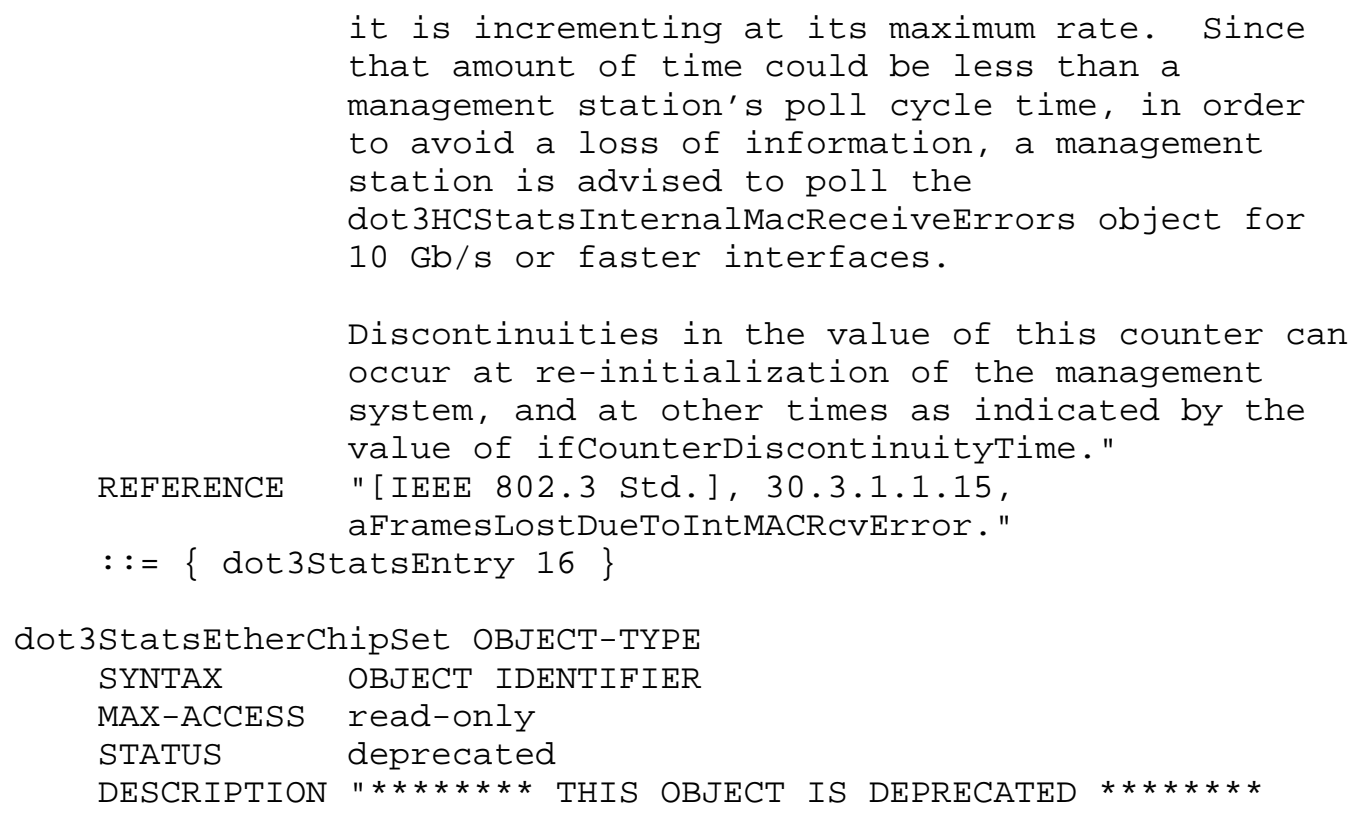

This object contains an OBJECT IDENTIFIER which identifies the chipset used to realize the interface. Ethernet-like interfaces are typically built out of several different chips. The MIB implementor is presented with a decision of which chip to identify via this object. The implementor should identify the chip which is usually called the Medium Access Control chip. If no such chip is easily identifiable, the implementor should identify the chip which actually gathers the transmit and receive statistics and error indications. This would allow a manager station to correlate the statistics and the chip generating them, giving it the ability to take into account any known anomalies in the chip.

This object has been deprecated. Implementation feedback indicates that it is of limited use for debugging network problems in the field, and the administrative overhead involved in maintaining a registry of chipset OIDs is not justified." 


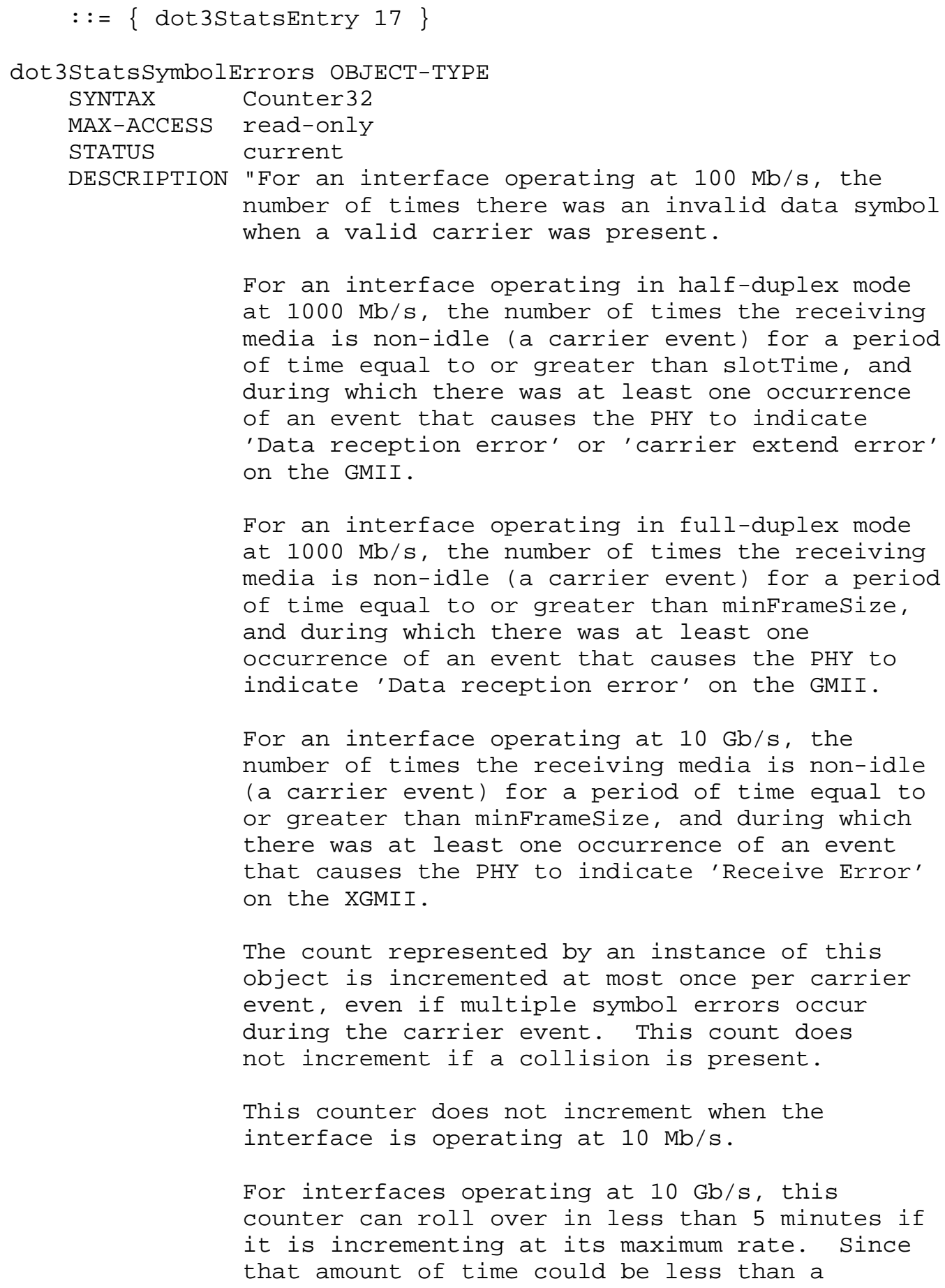




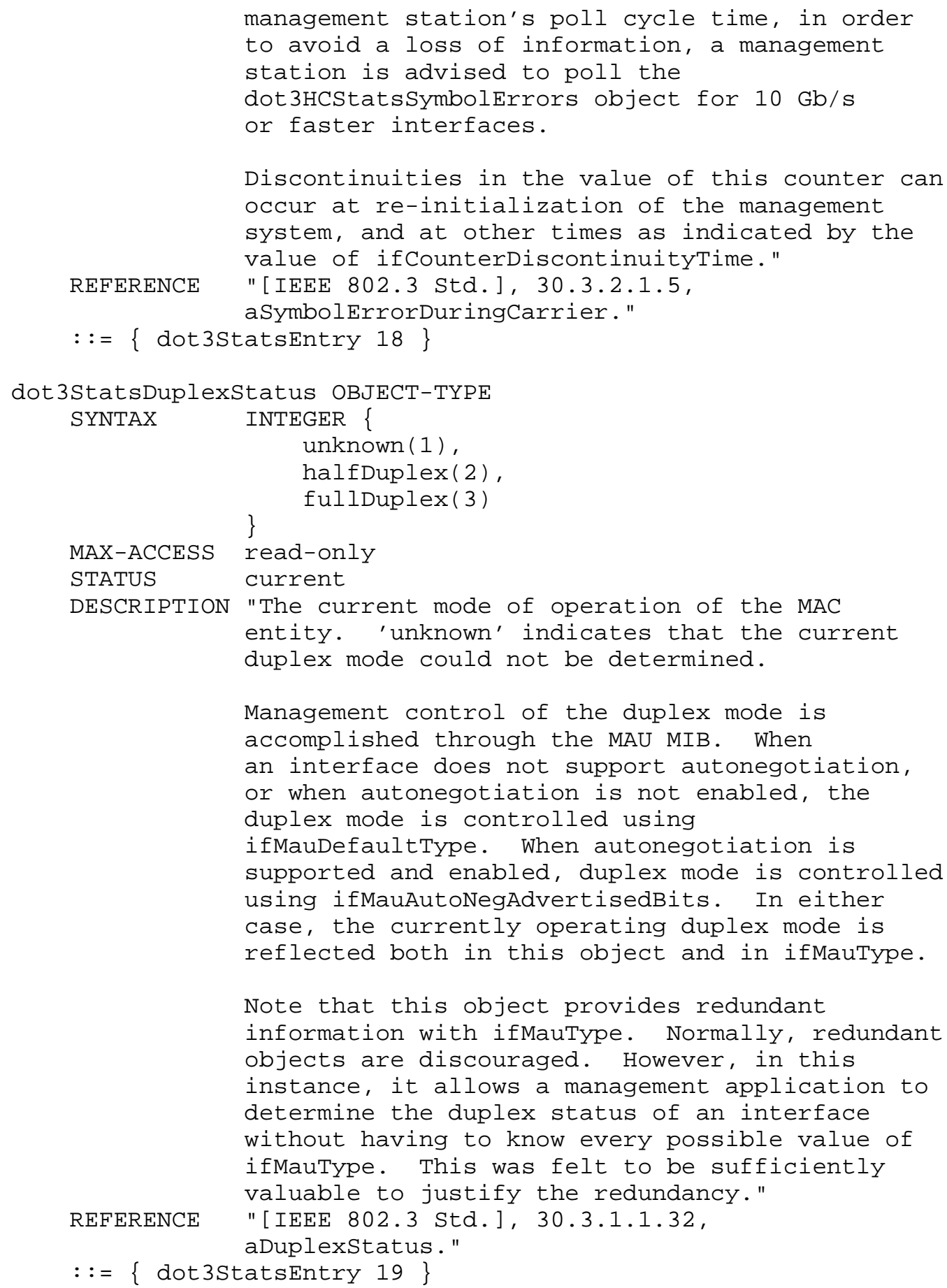

Flick 


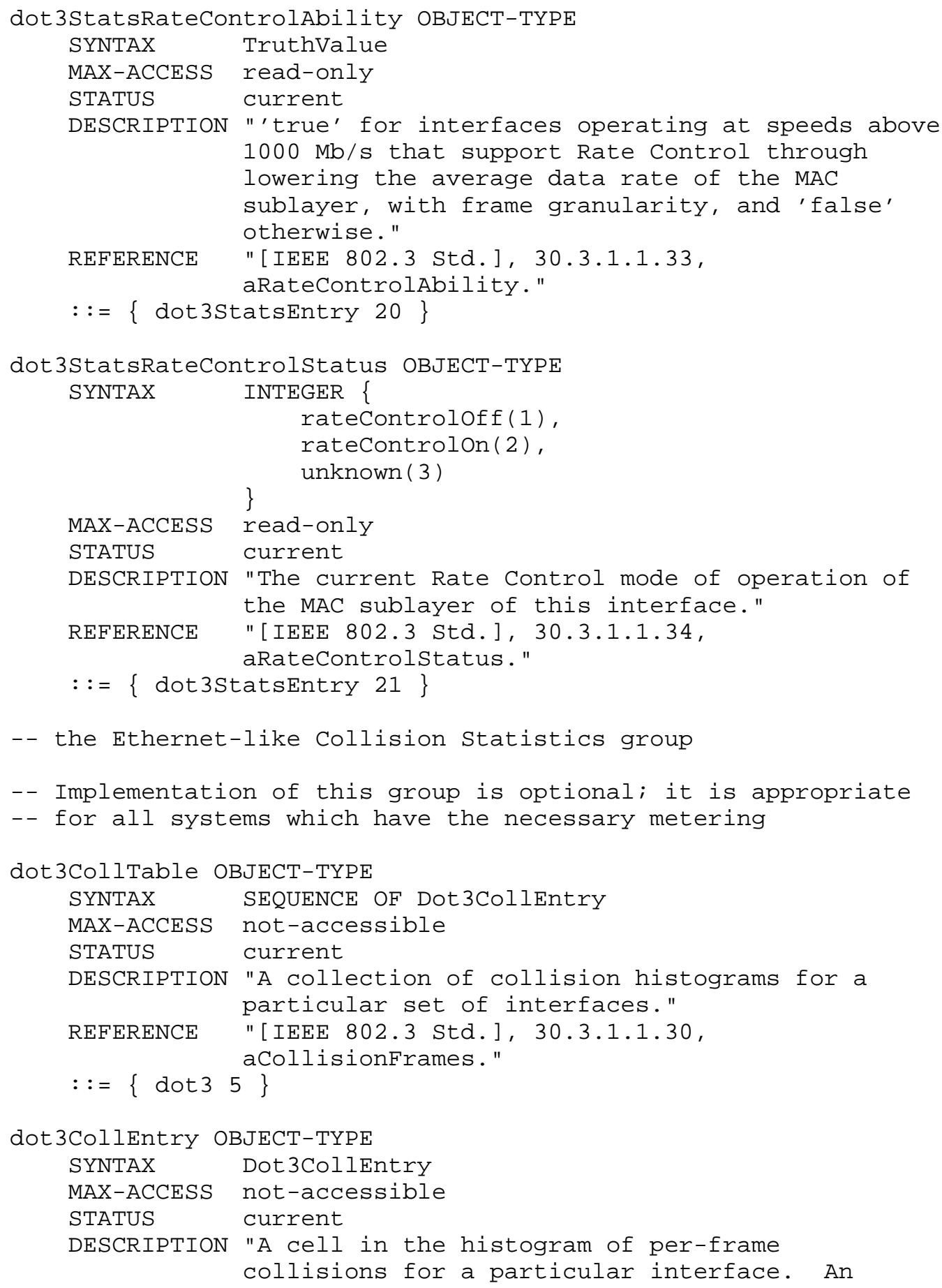

Flick 


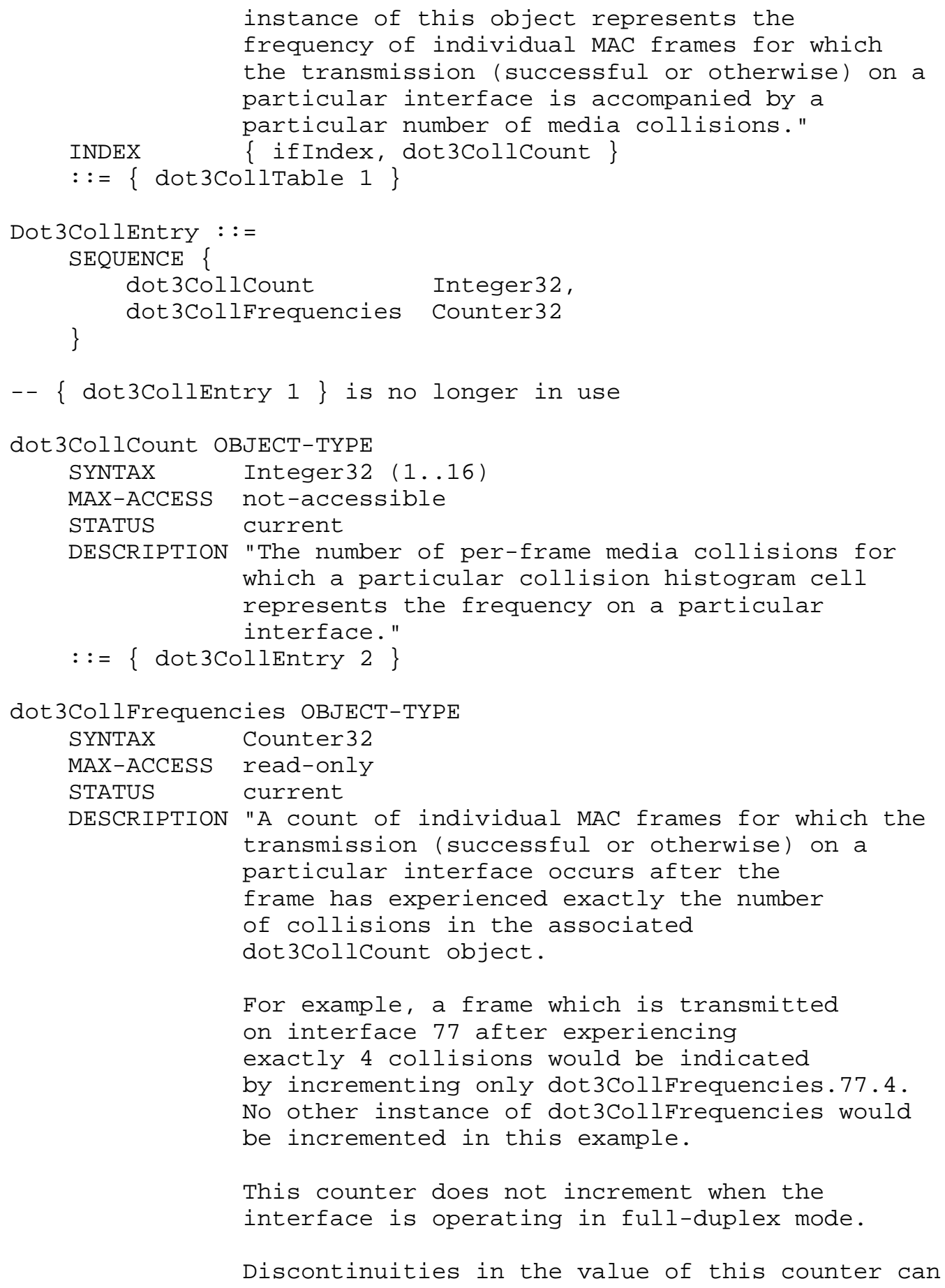




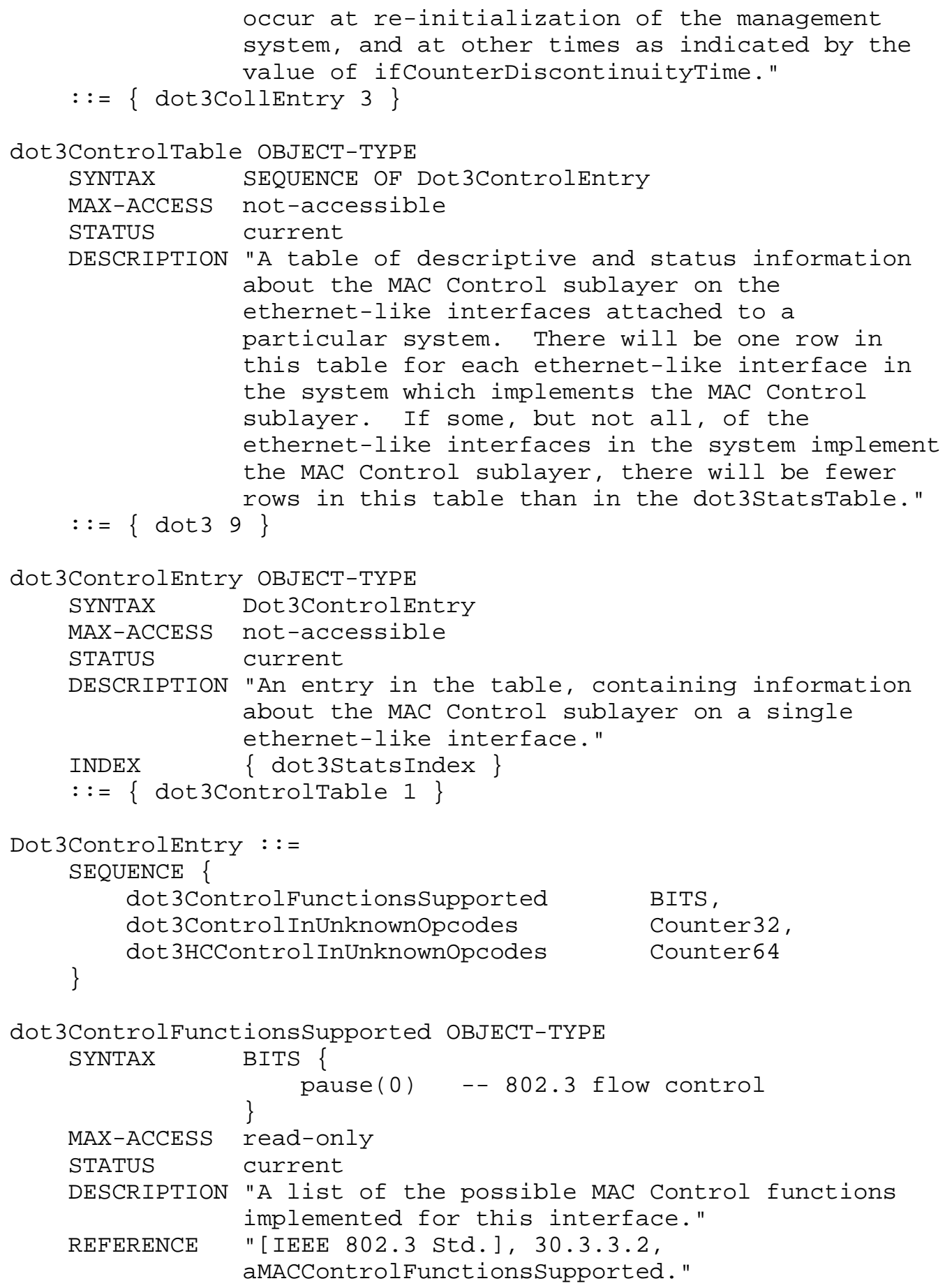

Flick 


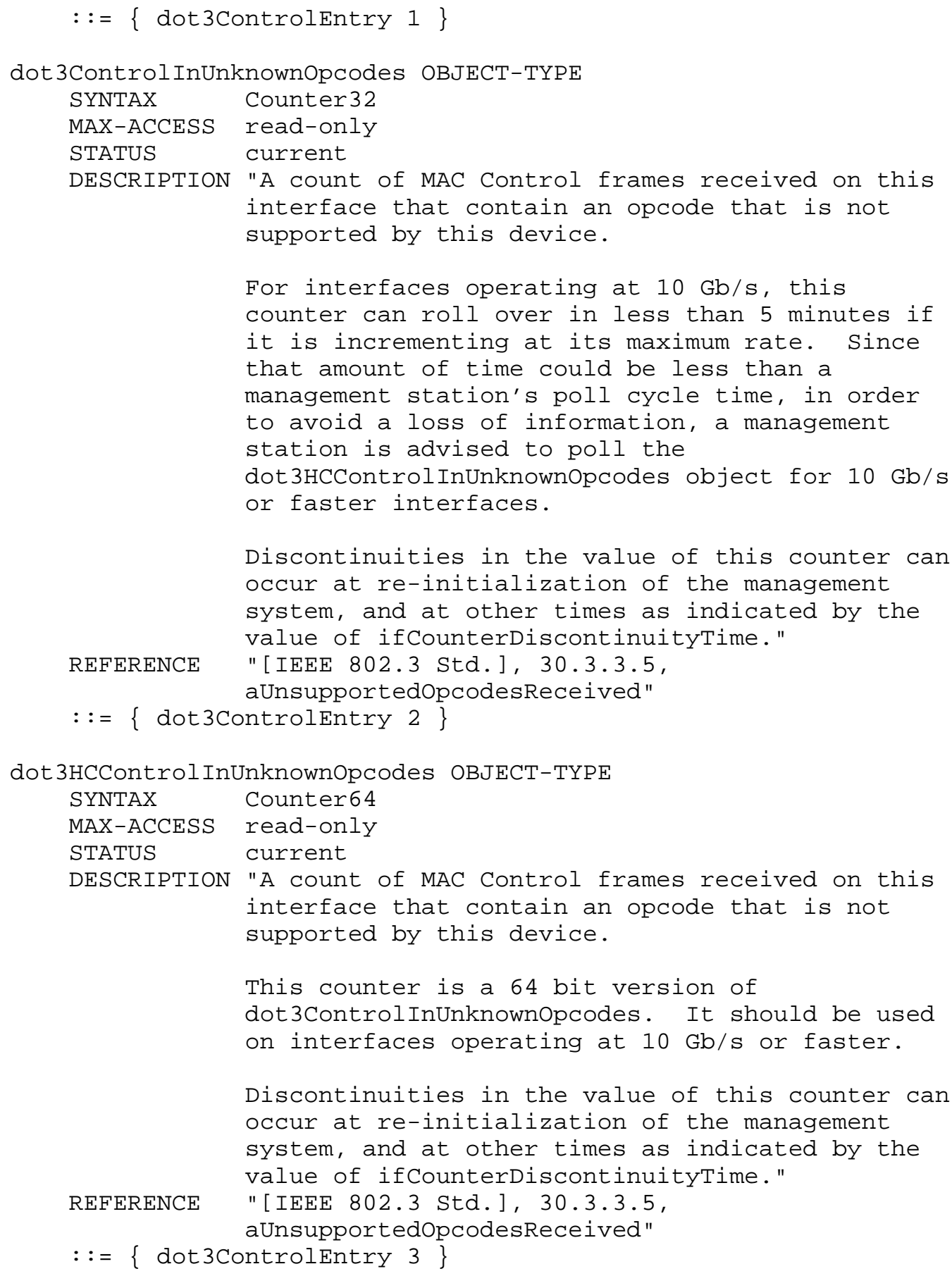




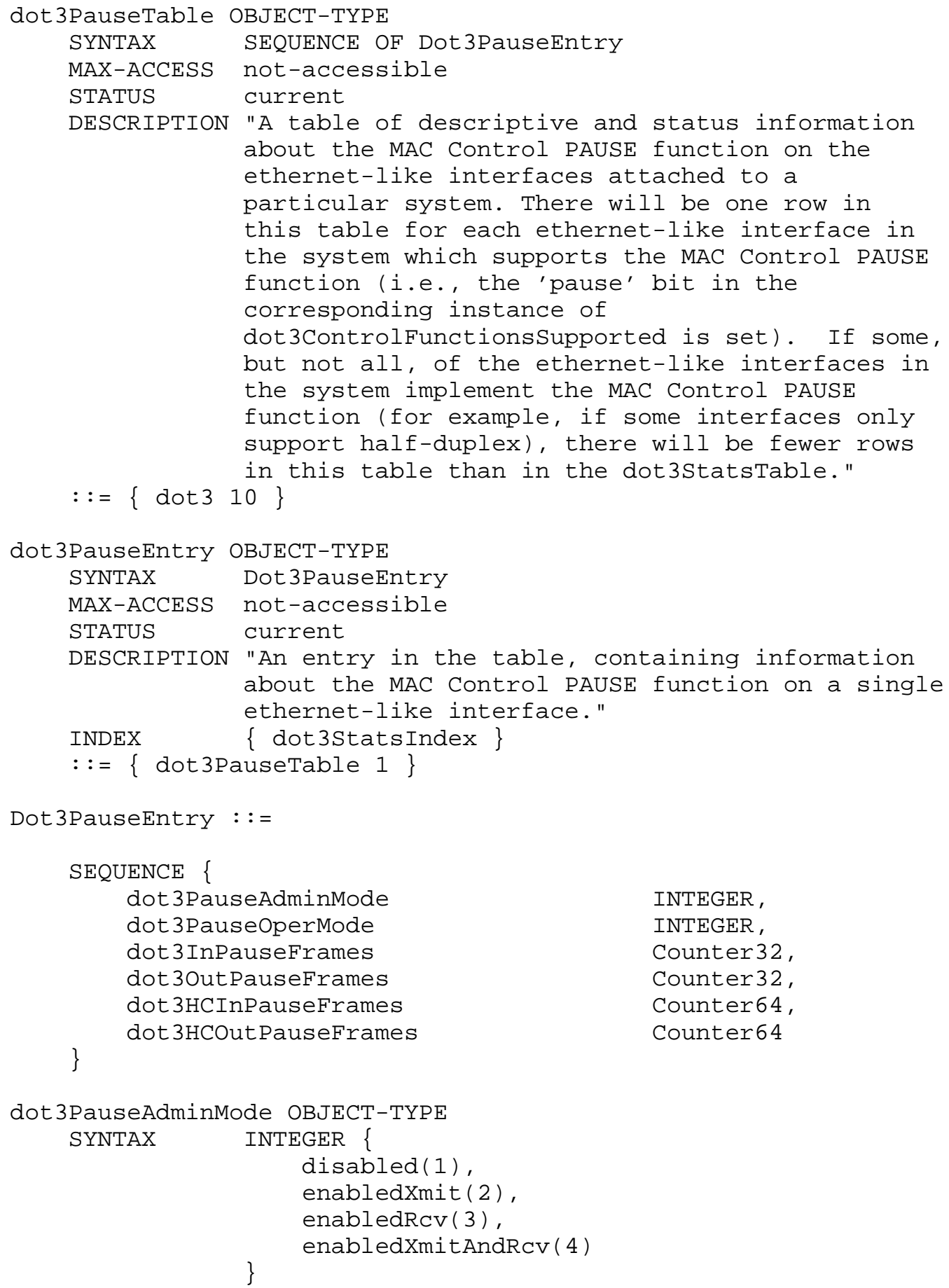

Flick 


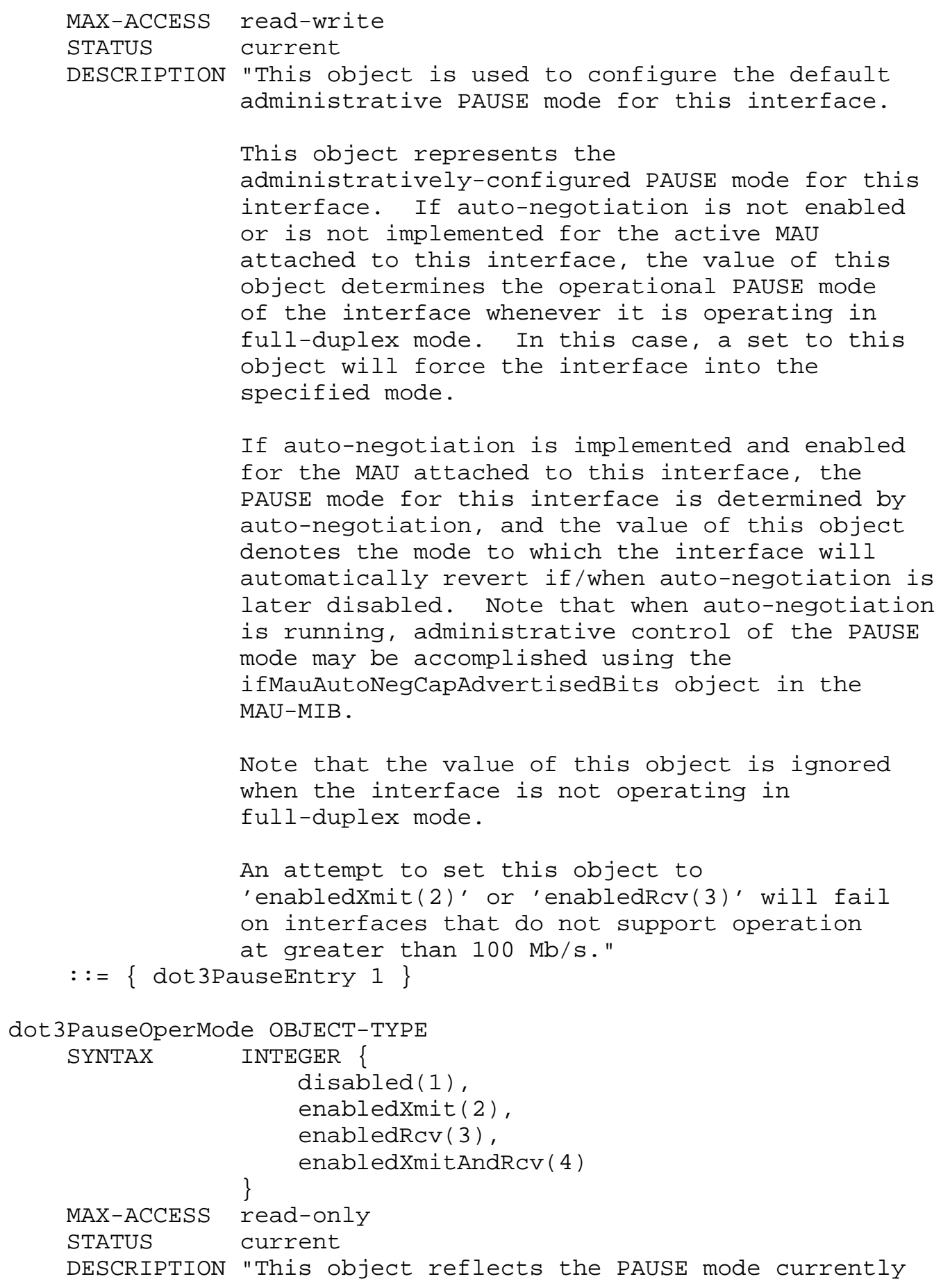




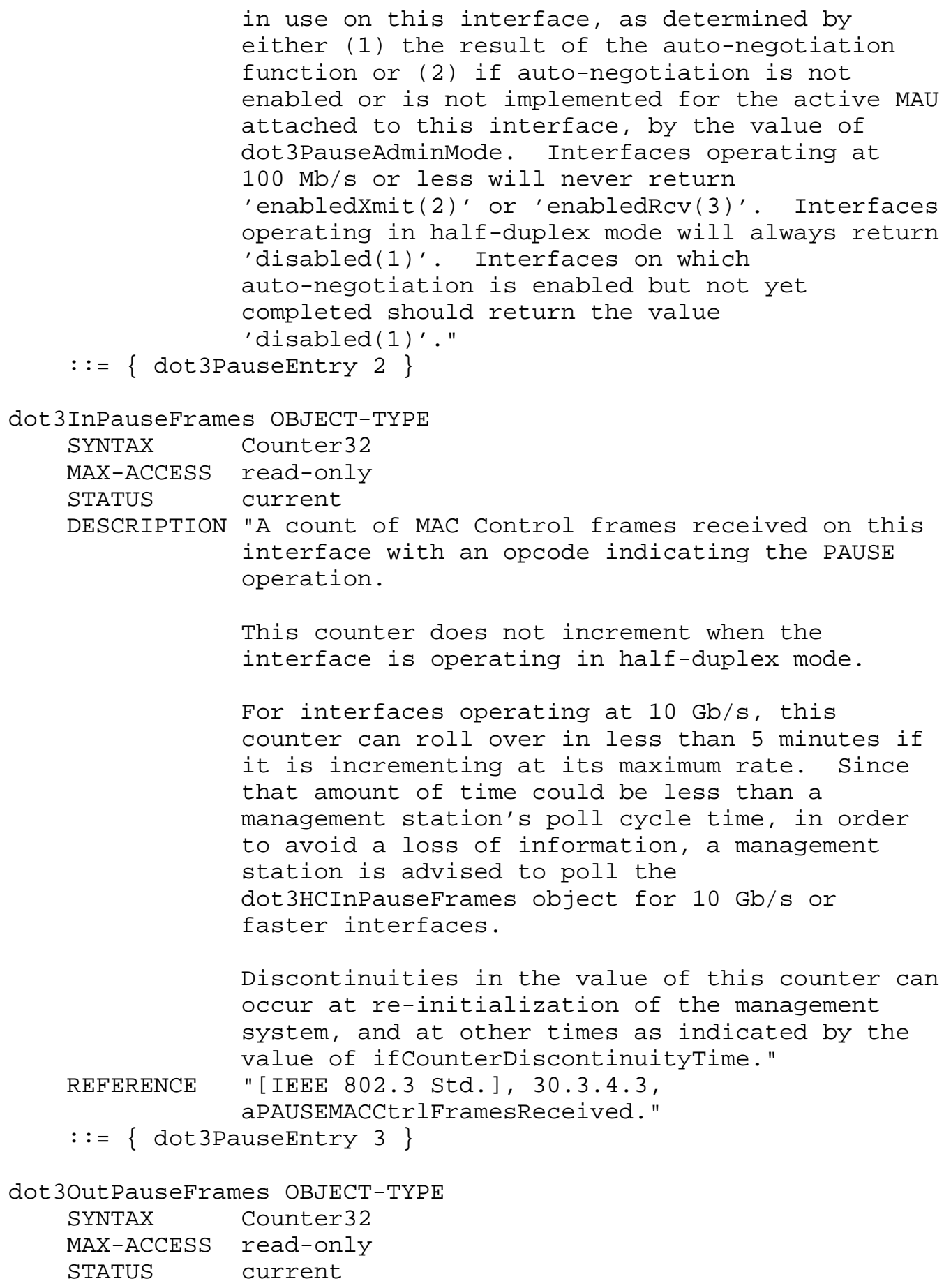




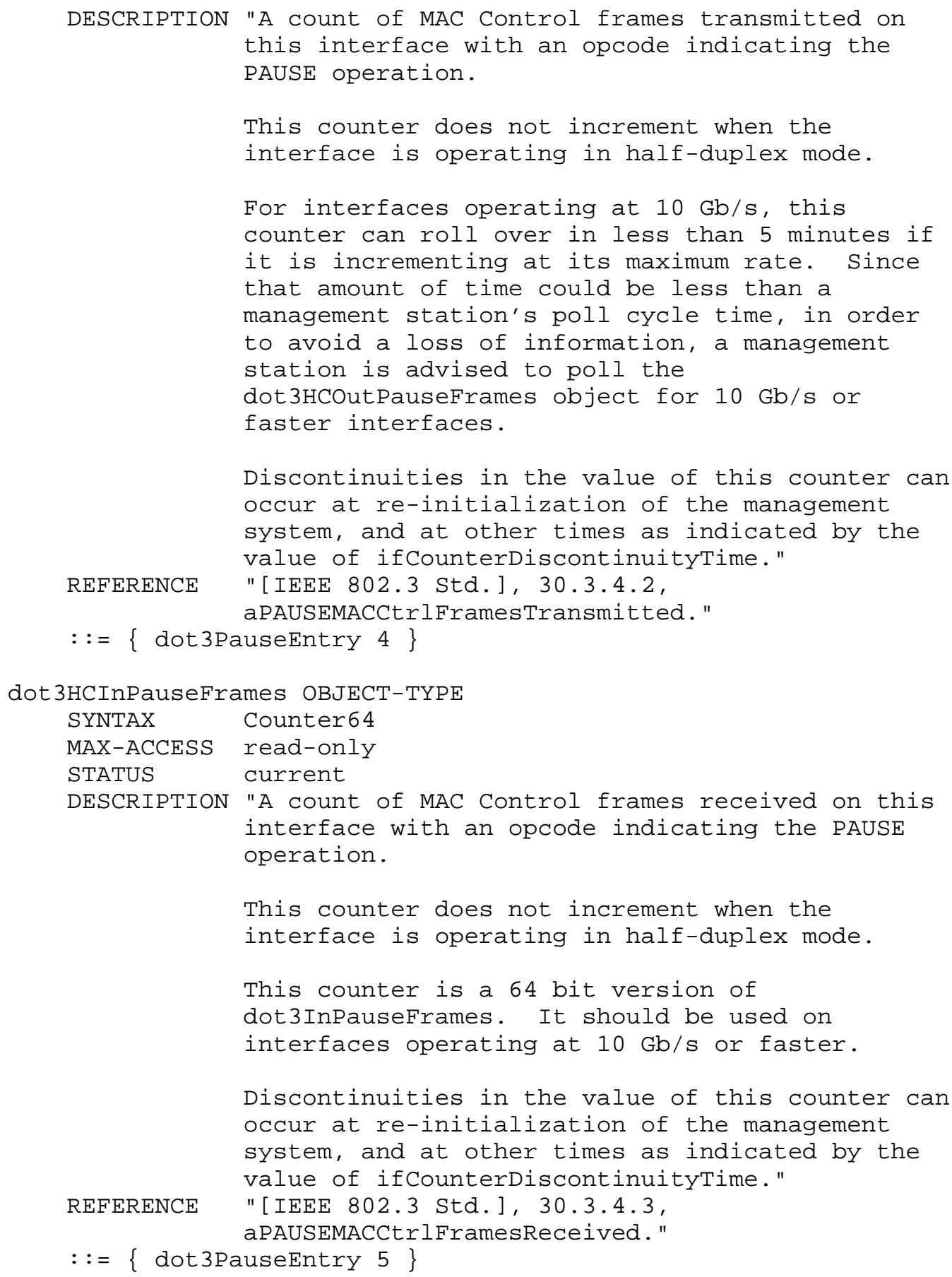




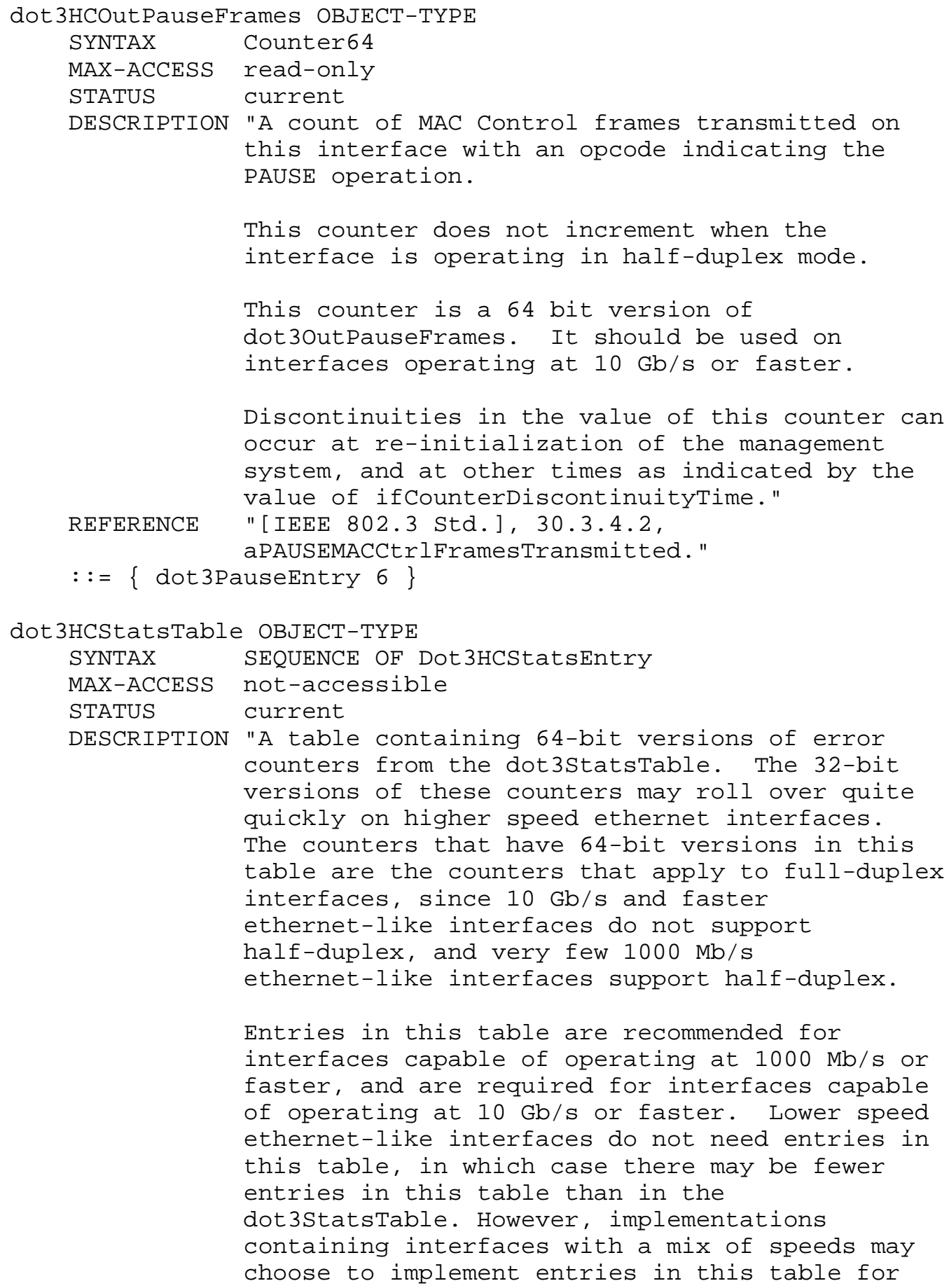




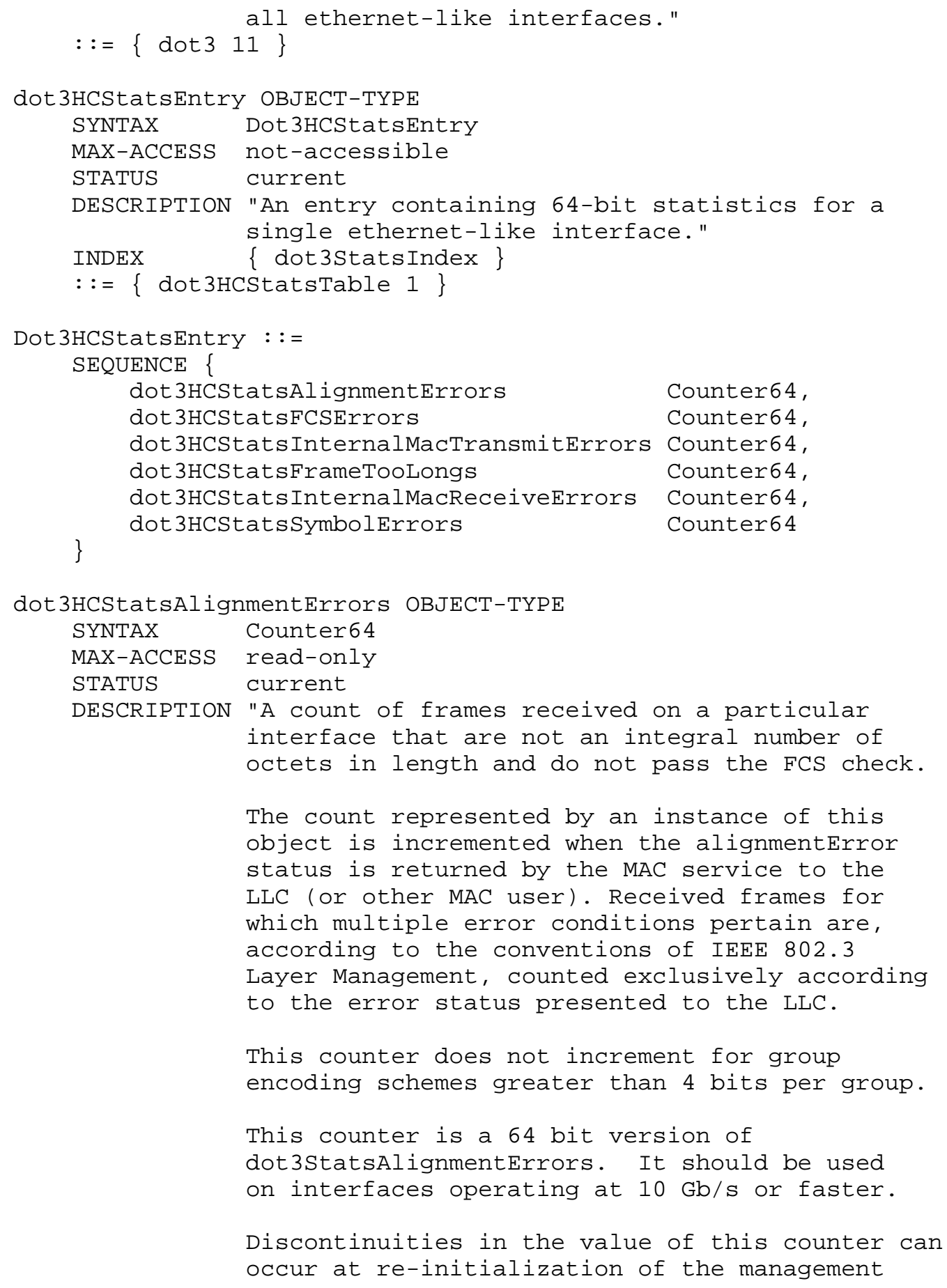




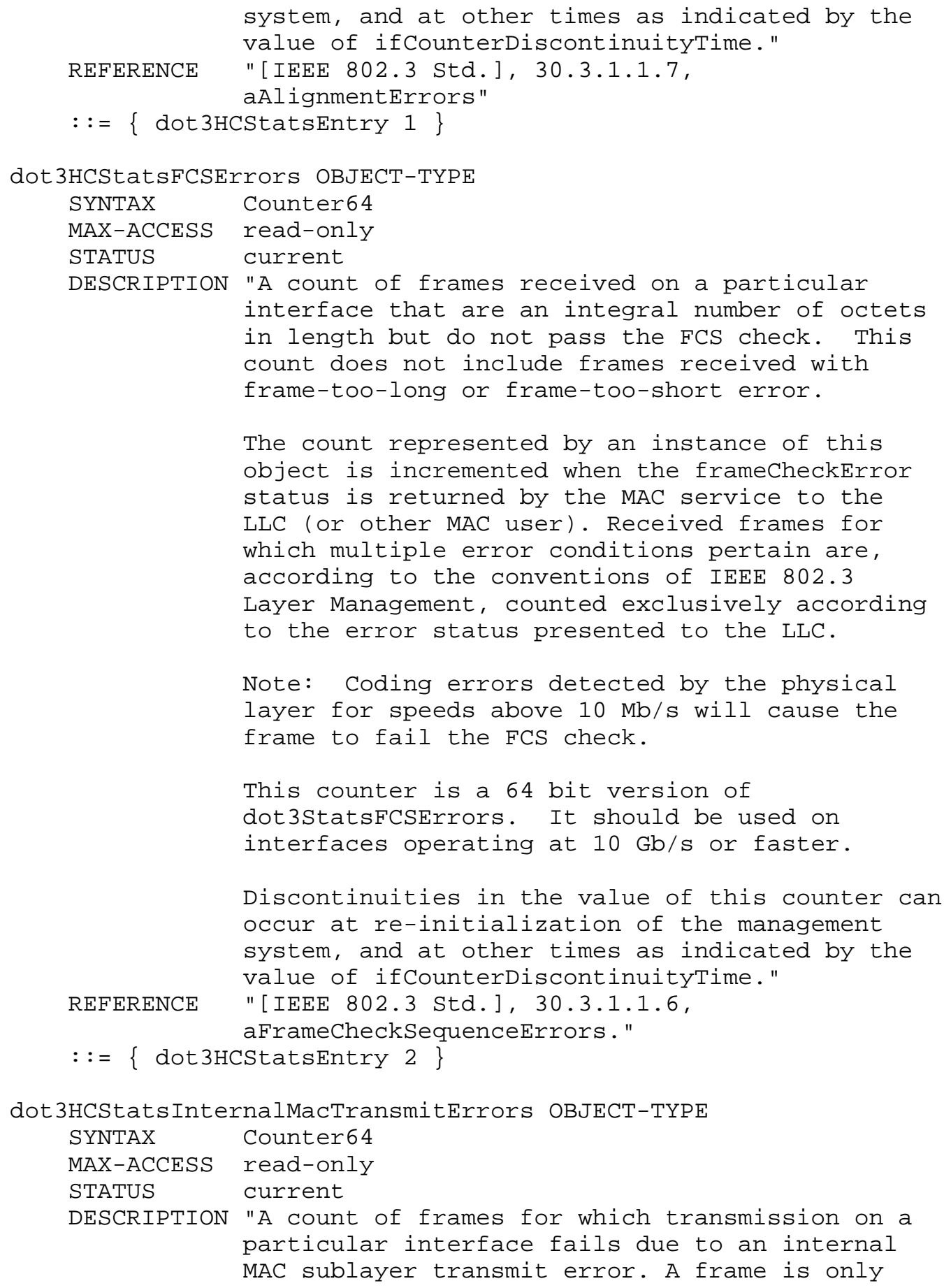




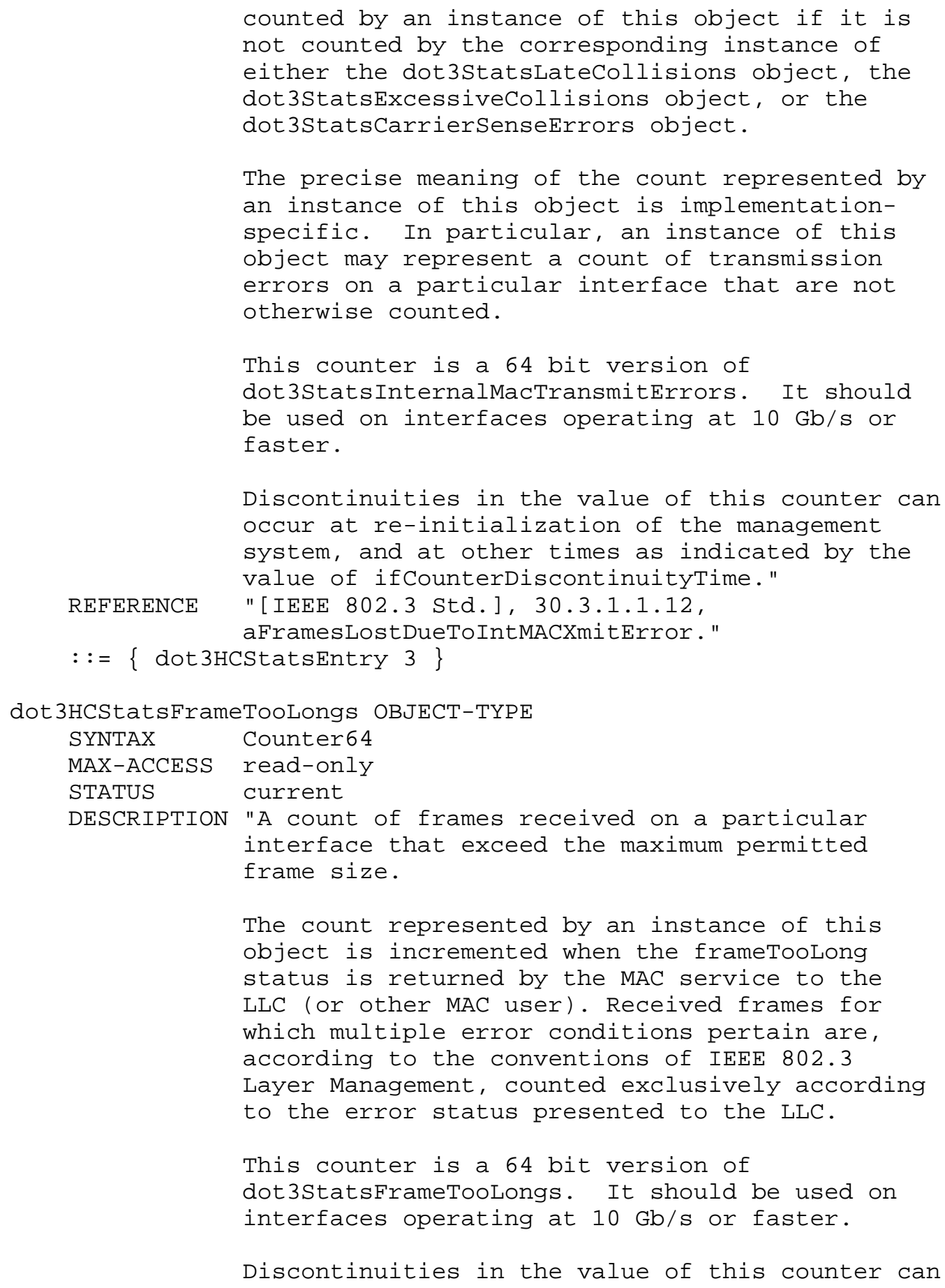




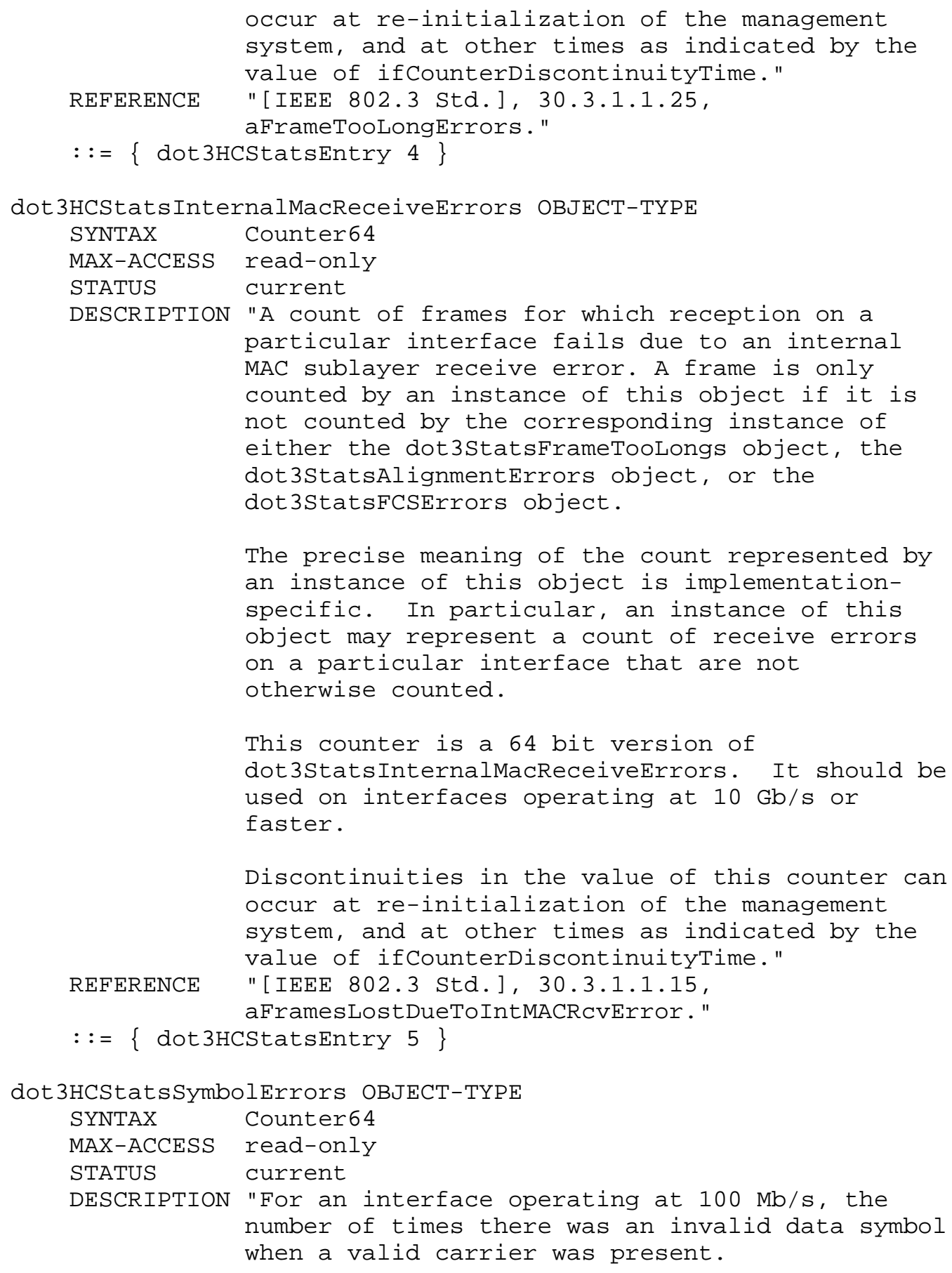




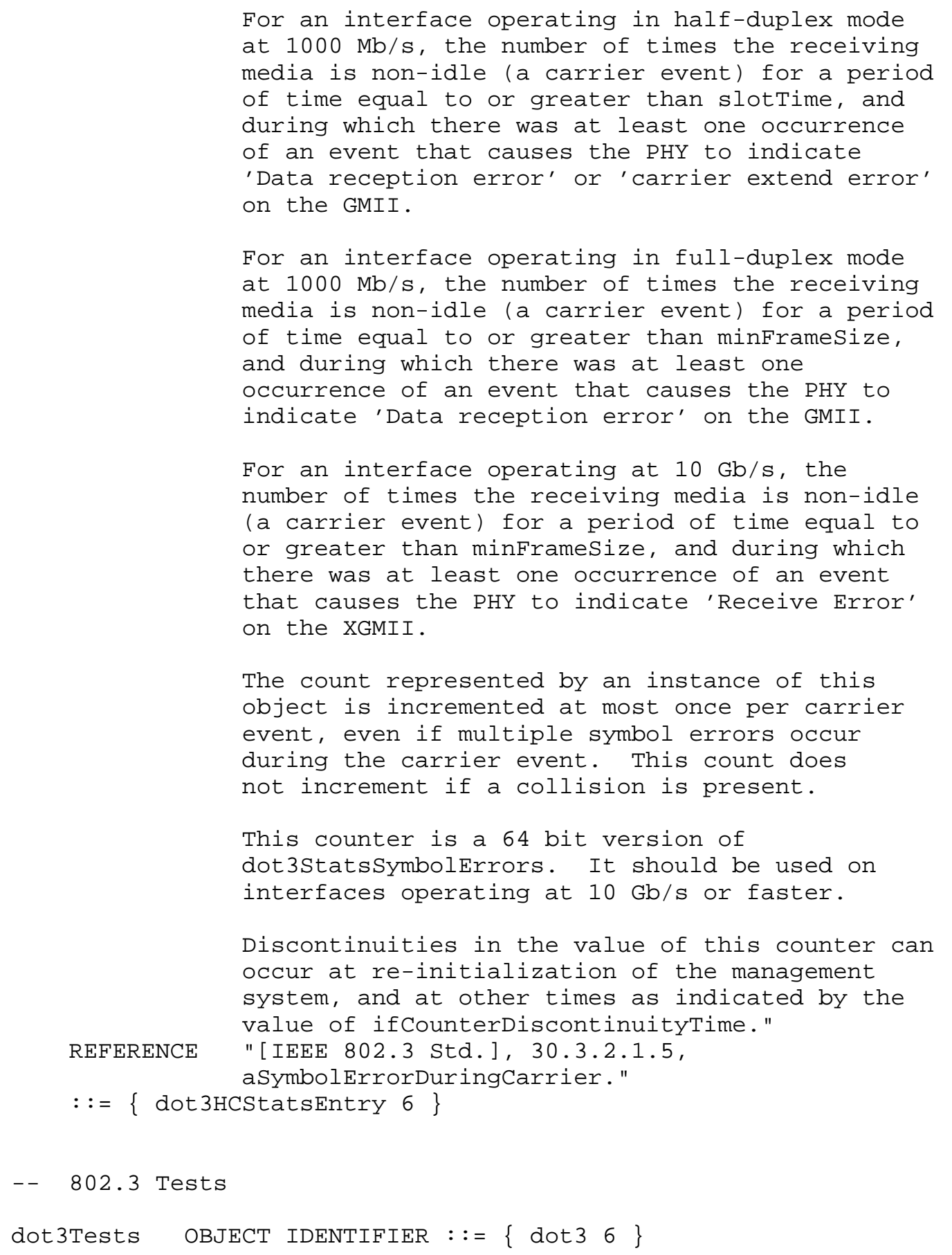




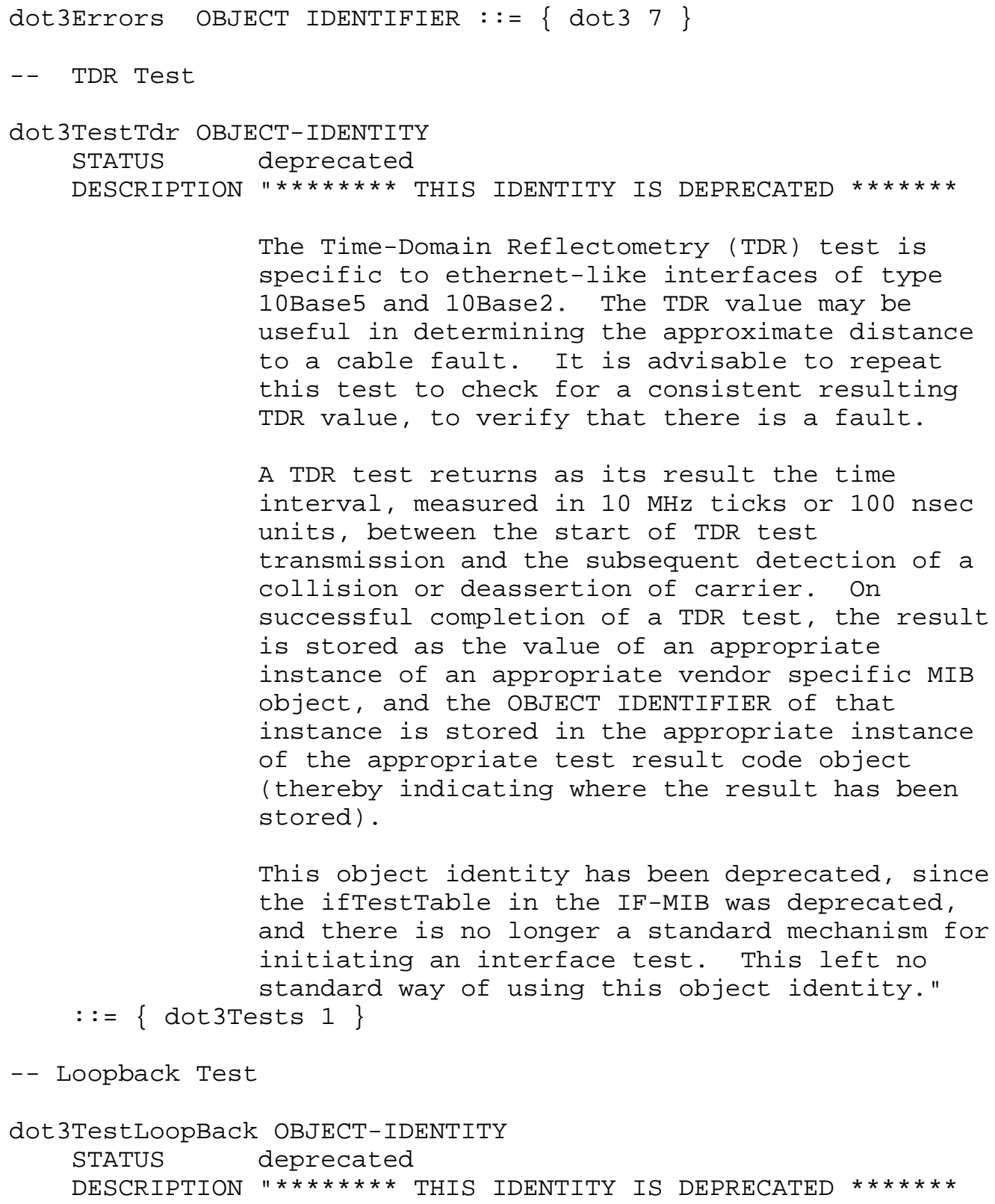

This test configures the MAC chip and executes an internal loopback test of memory, data paths, and the MAC chip logic. This loopback test can only be executed if the interface is offline. Once the test has completed, the MAC chip should 


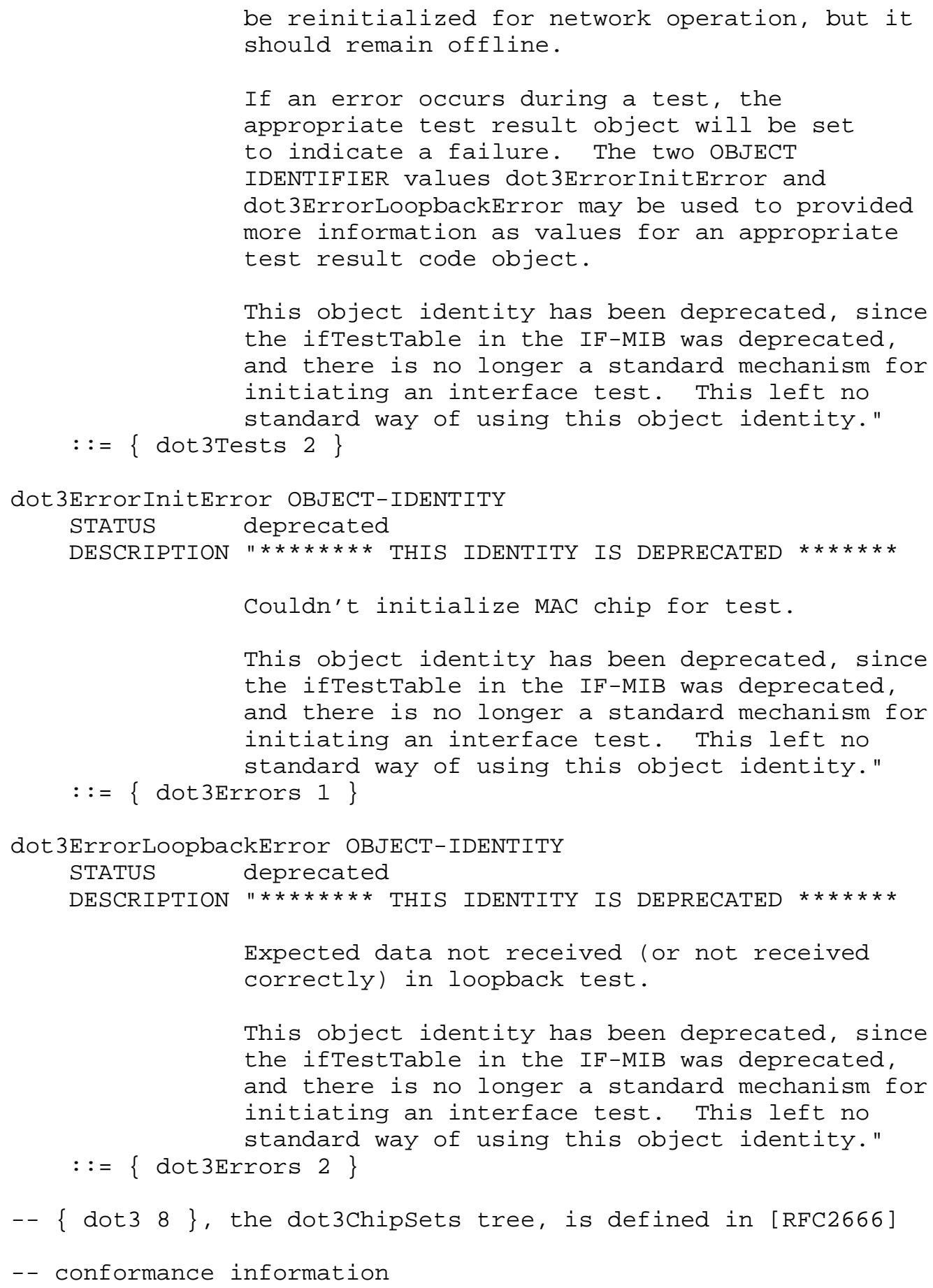




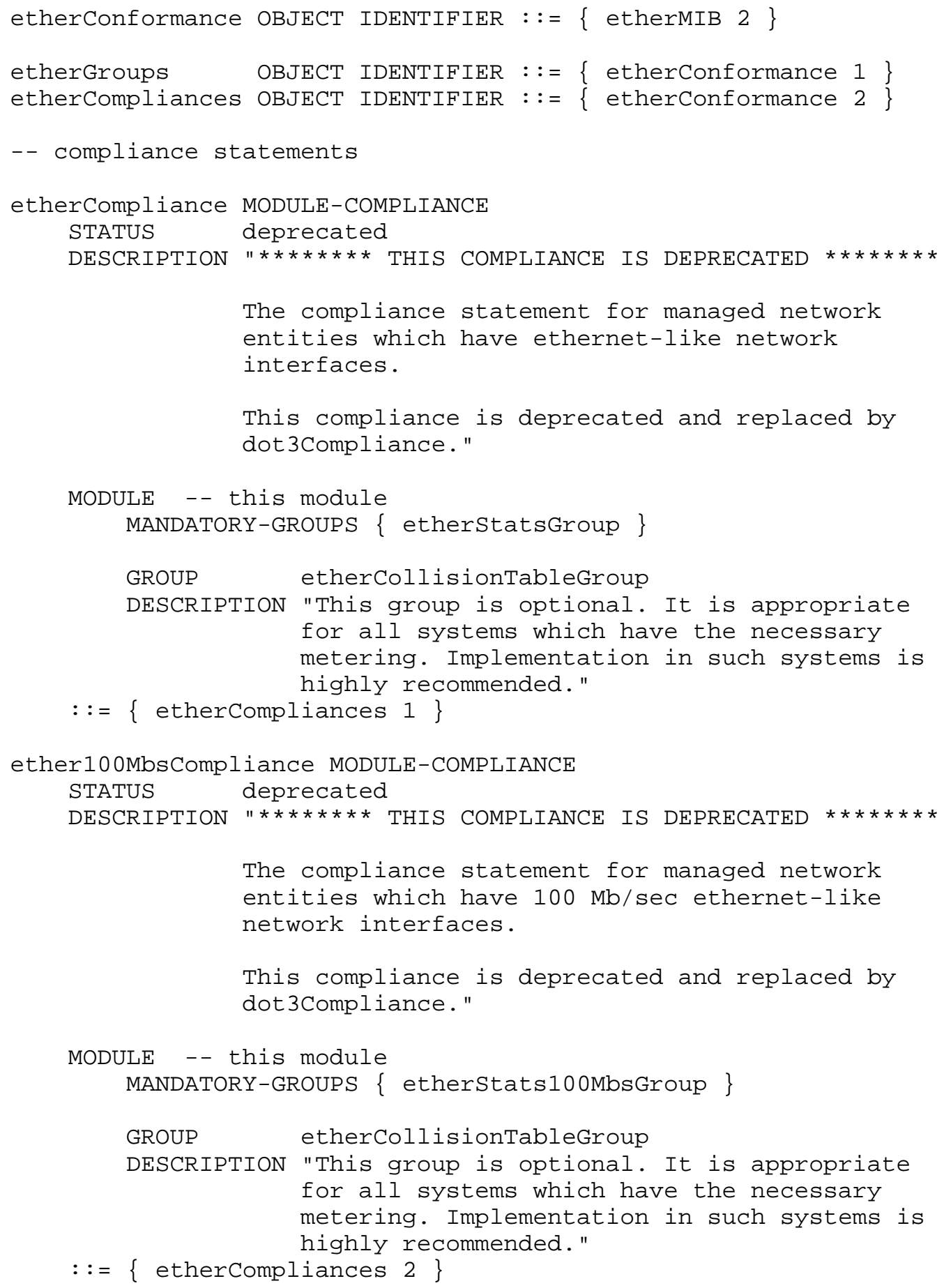

Flick 


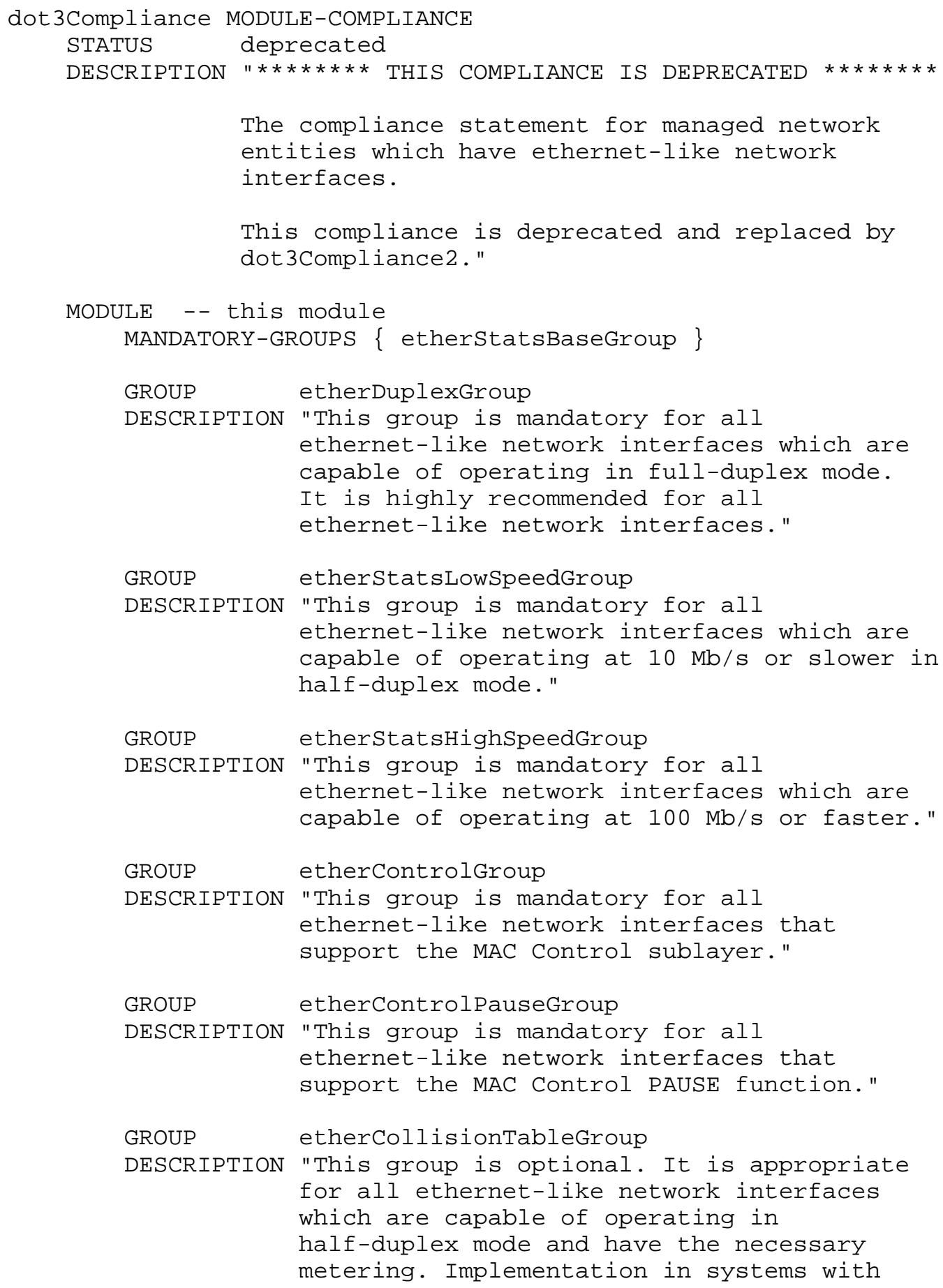

Flick 


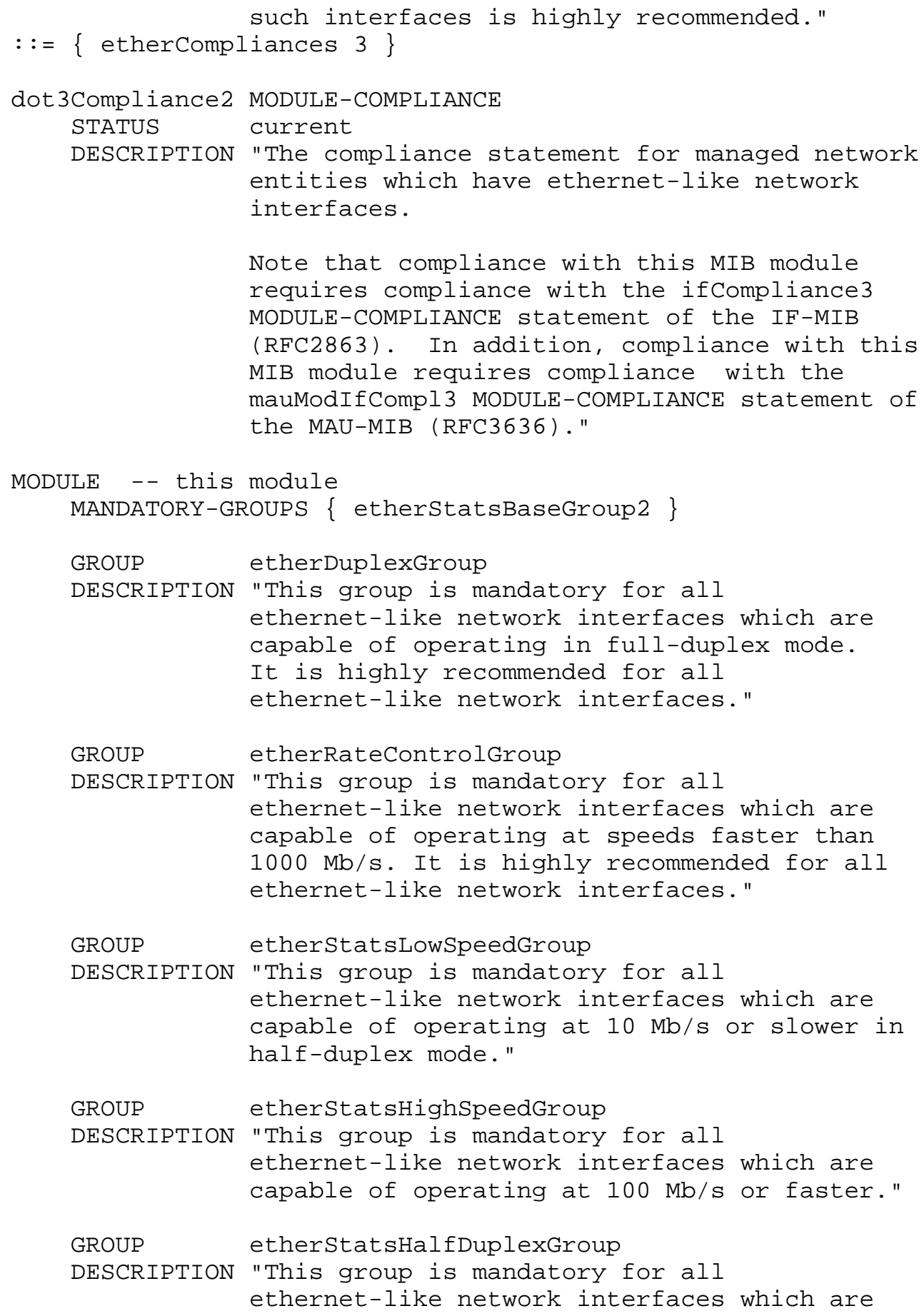

Flick 


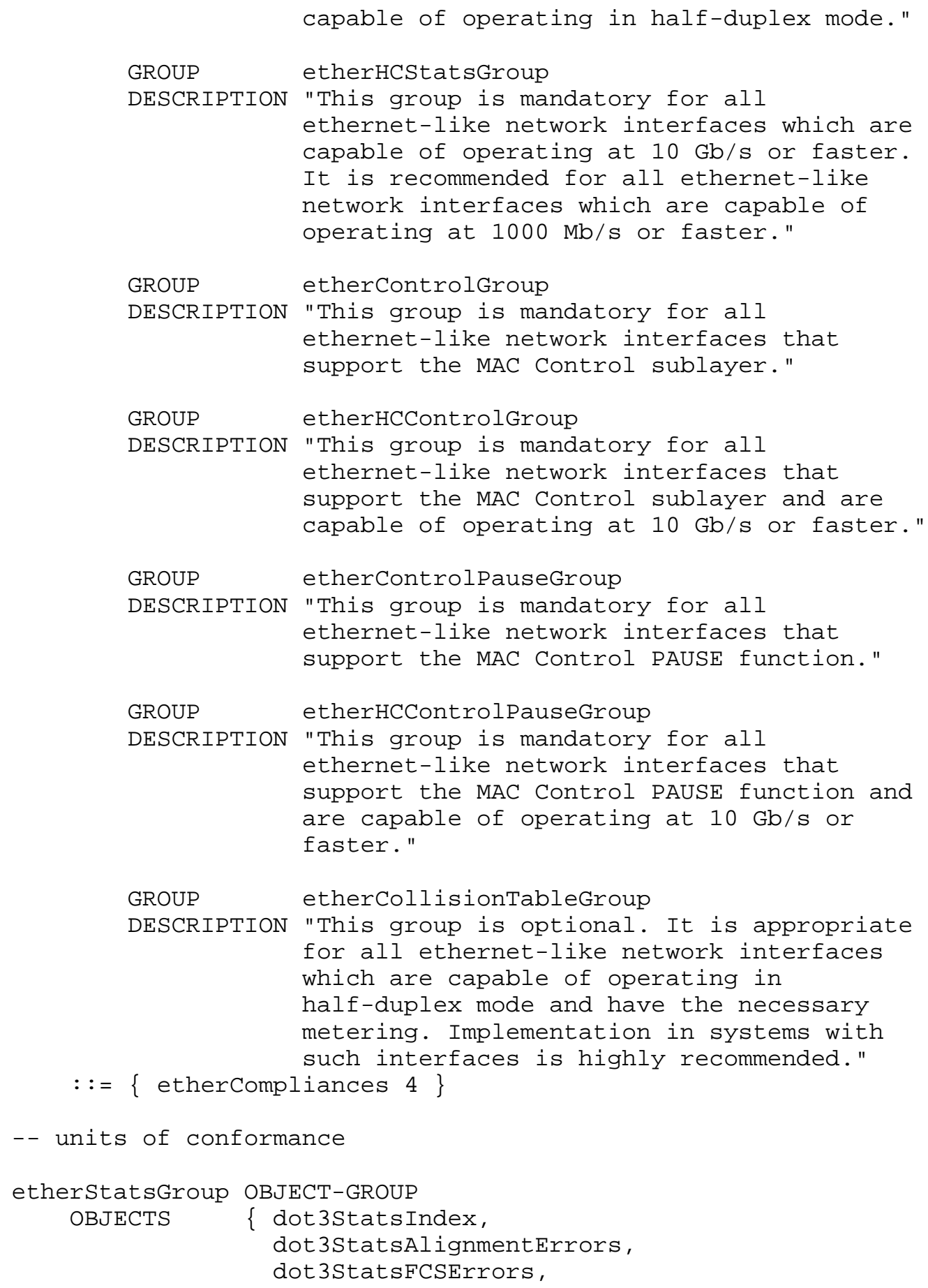




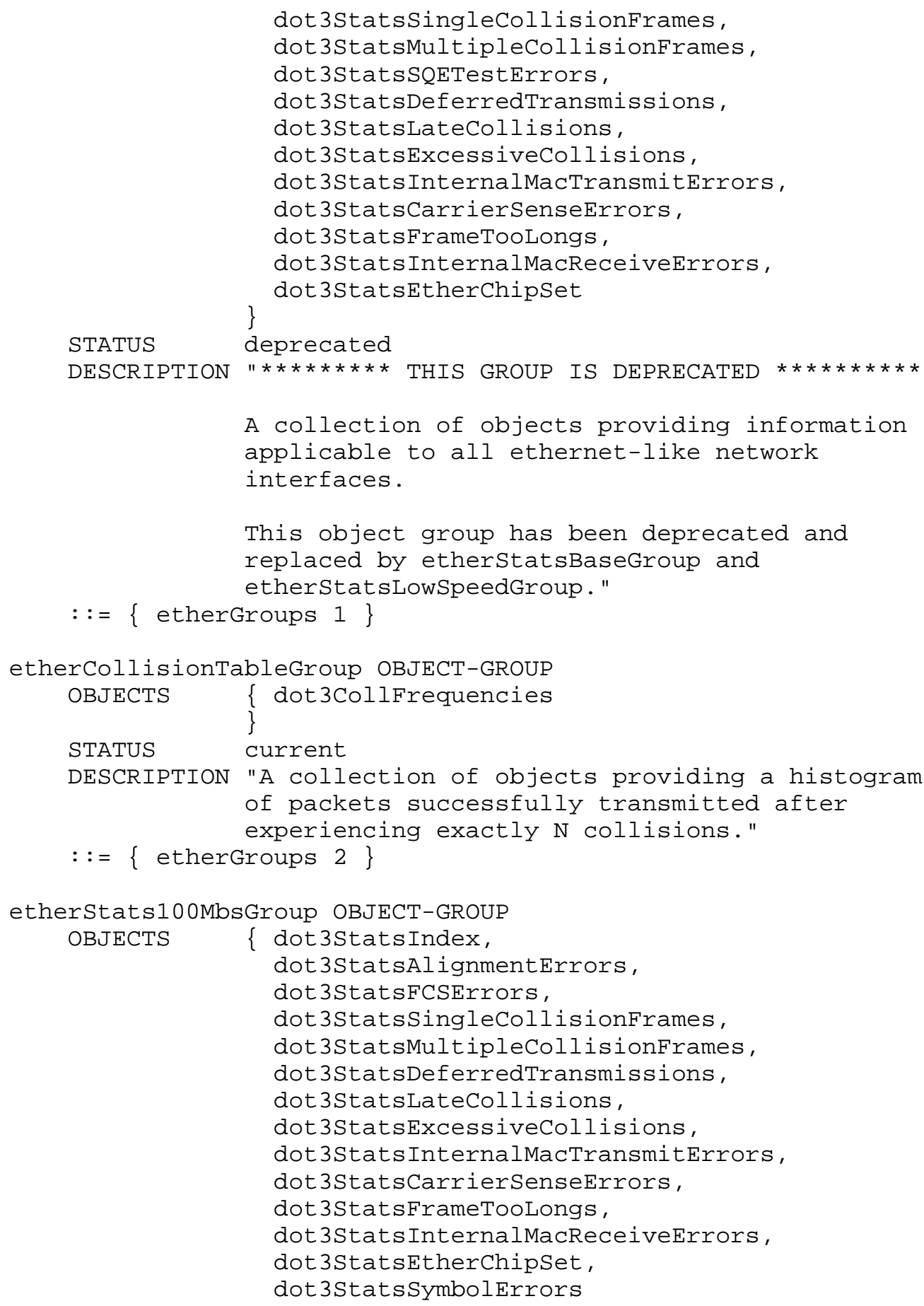

Flick 


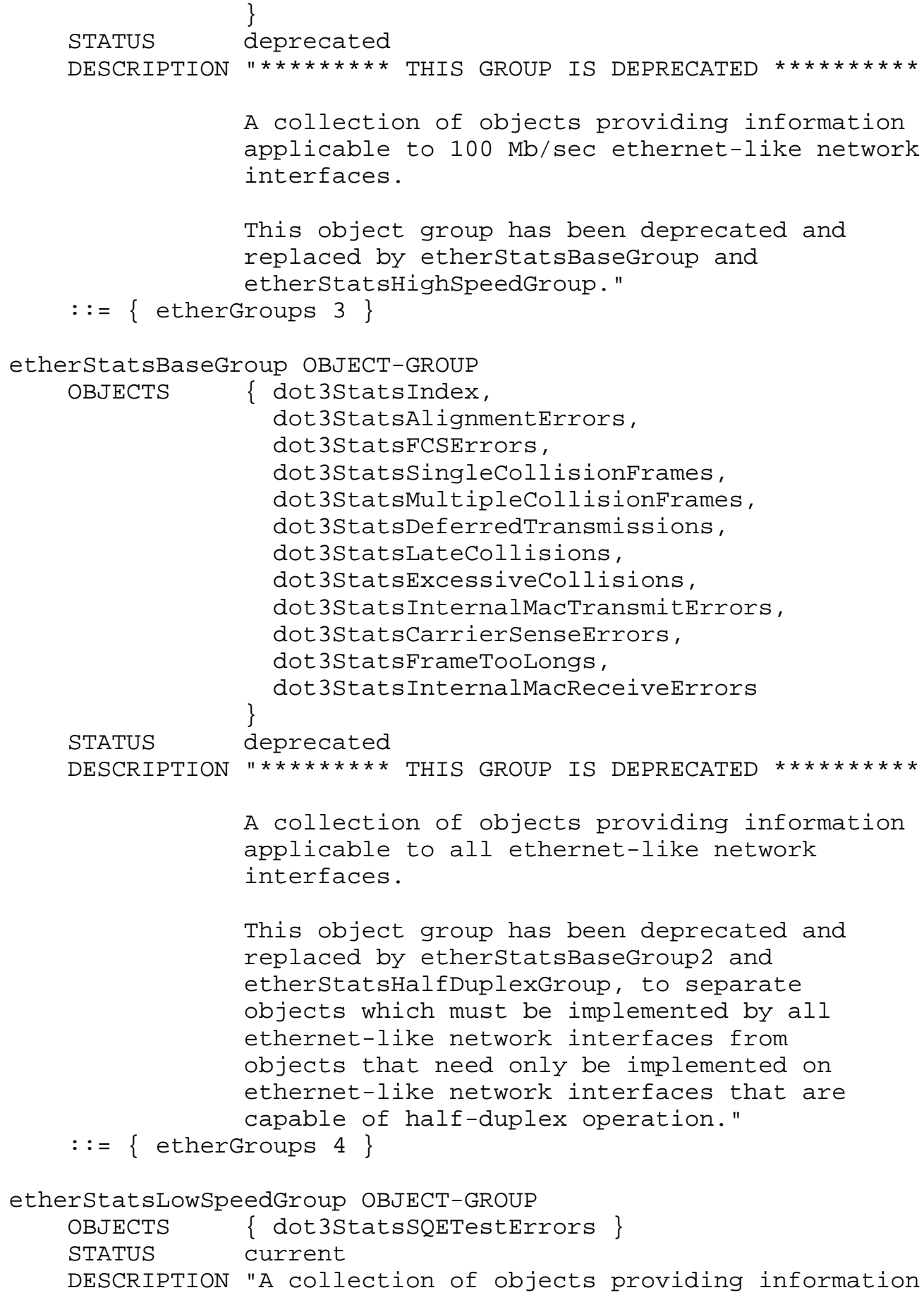




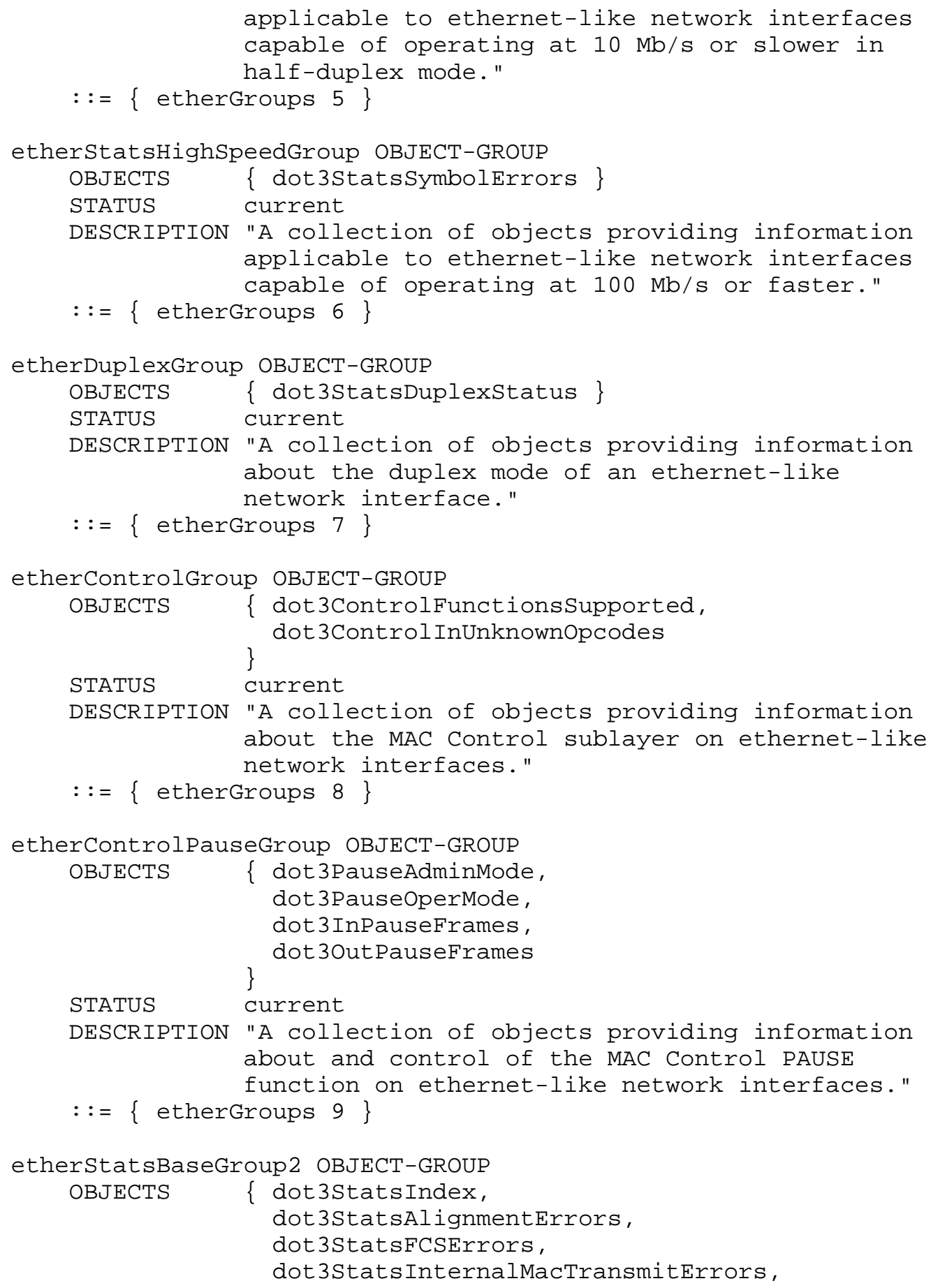

Flick 


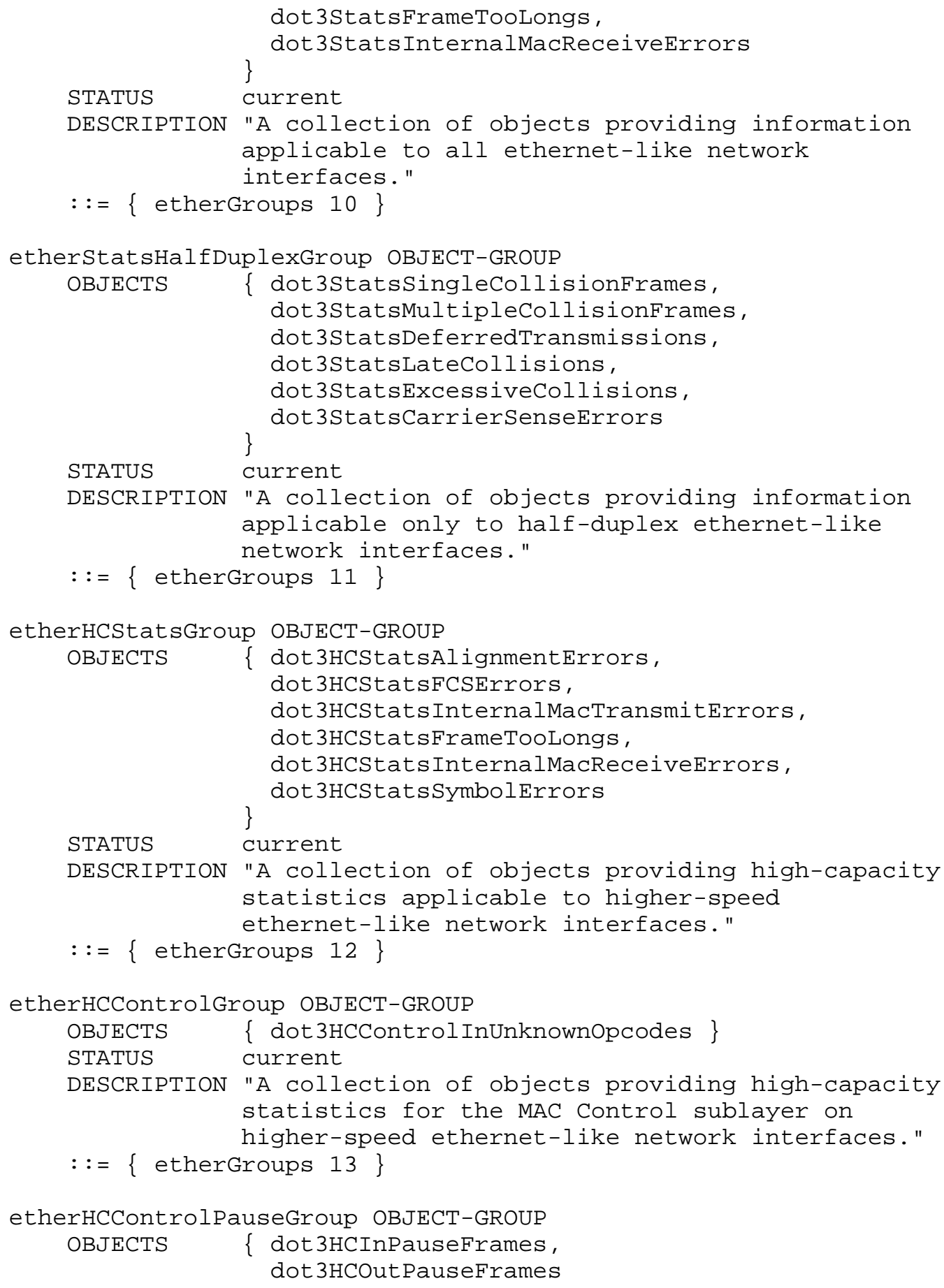

Flick 


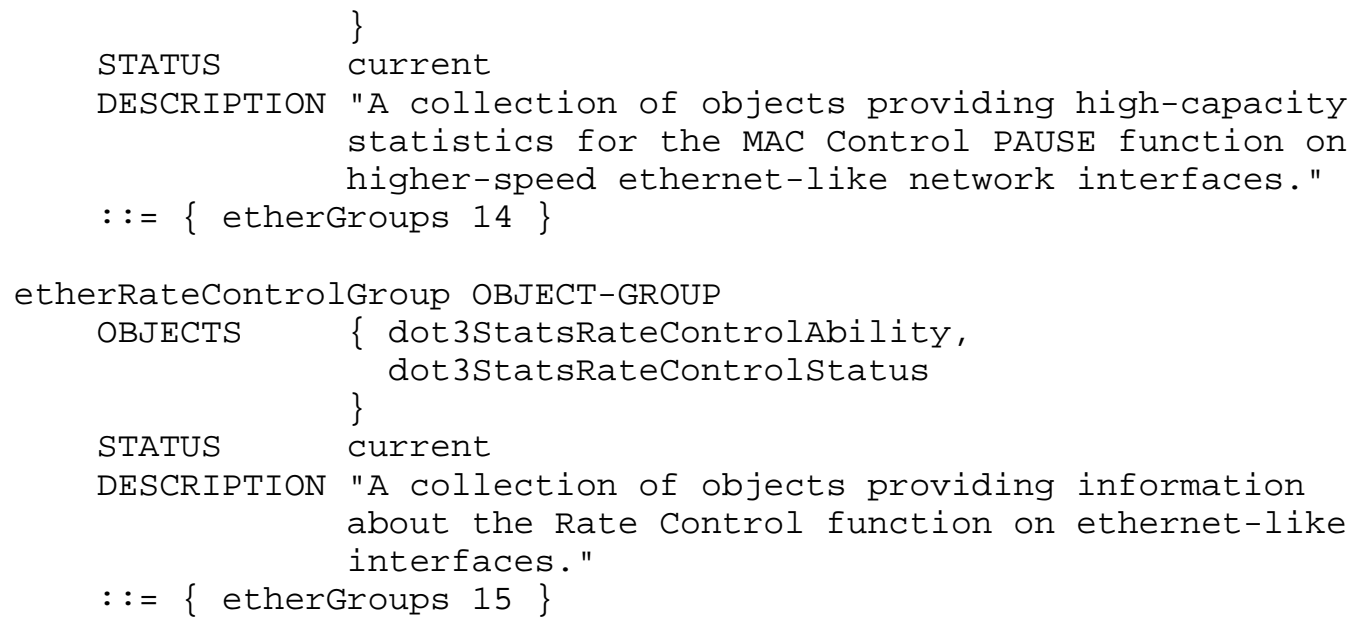

END

5. Intellectual Property statement

The IETF takes no position regarding the validity or scope of any intellectual property or other rights that might be claimed to pertain to the implementation or use of the technology described in this document or the extent to which any license under such rights might or might not be available; neither does it represent that it has made any effort to identify any such rights. Information on the IETF's procedures with respect to rights in standards-track and standards-related documentation can be found in BCP-11. Copies of claims of rights made available for publication and any assurances of licenses to be made available, or the result of an attempt made to obtain a general license or permission for the use of such proprietary rights by implementors or users of this specification can be obtained from the IETF secretariat.

The IETF invites any interested party to bring to its attention any copyrights, patents or patent applications, or other proprietary rights which may cover technology that may be required to practice this standard. Please address the information to the IETF Executive Director. 


\title{
6. Acknowledgements
}

This document was produced by the IETF Ethernet Interfaces and Hub MIB Working Group, whose efforts were greatly advanced by the contributions of the following people:

\author{
Ran Atkinson \\ Mike Ayers \\ Mike Heard \\ Jeffrey Johnson \\ Lynn Kubinec \\ Kam Lam \\ Kerry McDonald \\ Steve McRobert \\ K.C. Norseth \\ Dan Romascanu \\ Randy Presuhn \\ Andrew Smith \\ Kaj Tesink \\ Geoff Thompson
}

This document is based on the Proposed Standard Ethernet MIB, RFC 2665 [RFC2665], edited by John Flick of Hewlett-Packard and Jeffrey Johnson of RedBack Networks and produced by the Ethernet Interfaces and Hub MIB Working Group. It extends that document by providing support for $10 \mathrm{~Gb} / \mathrm{s}$ Ethernet interfaces as defined in [IEEE802.3ae].

RFC 2665, in turn, is based on the Proposed Standard Ethernet MIB, RFC 2358 [RFC2358], edited by John Flick of Hewlett-Packard and Jeffrey Johnson of RedBack Networks and produced by the 802.3 Hub MIB Working Group. It extends that document by providing support for full-duplex Ethernet interfaces and $1000 \mathrm{Mb} / \mathrm{sec}$ Ethernet interfaces as outlined in [IEEE802.3].

RFC 2358, in turn, is almost completely based on both the Standard Ethernet MIB, RFC 1643 [RFC1643], and the Proposed Standard Ethernet MIB using the SNMPv2 SMI, RFC 1650 [RFC1650], both of which were edited by Frank Kastenholz of FTP Software and produced by the Interfaces MIB Working Group. RFC 2358 extends those documents by providing support for $100 \mathrm{Mb} / \mathrm{sec}$ ethernet interfaces.

RFC 1643 and RFC 1650, in turn, are based on the Draft Standard Ethernet MIB, RFC 1398 [RFC1398], also edited by Frank Kastenholz and produced by the Ethernet MIB Working Group. 
RFC 1398, in turn, is based on the Proposed Standard Ethernet MIB, RFC 1284 [RFC1284], which was edited by John Cook of Chipcom and produced by the Transmission MIB Working Group. The Ethernet MIB Working Group gathered implementation experience of the variables specified in RFC 1284, documented that experience in RFC 1369 [RFC1369], and used that information to develop this revised MIB.

RFC 1284, in turn, is based on a document written by Frank Kastenholz, then of Interlan, entitled IEEE 802.3 Layer Management Draft M compatible MIB for TCP/IP Networks [KASTEN]. This document was modestly reworked, initially by the SNMP Working Group, and then by the Transmission Working Group, to reflect the current conventions for defining objects for MIB interfaces. James Davin, of the MIT Laboratory for Computer Science, and Keith McCloghrie of Hughes LAN Systems, contributed to later drafts of this memo. Marshall Rose of Performance Systems International, Inc. converted the document into RFC 1212 [RFC1212] concise format. Anil Rijsinghani of DEC contributed text that more adequately describes the TDR test. Thanks to Frank Kastenholz of Interlan and Louis Steinberg of IBM for their experimentation.

7. Normative References

[RFC2119] Bradner, S., "Key words for use in RFCs to Indicate Requirements Levels", BCP 14, RFC 2119, March 1997.

[RFC2578] MCCloghrie, K., Perkins, D., Schoenwaelder, J., Case, J., Rose, M. and S. Waldbusser, "Structure of Management Information Version 2 (SMIV2)", STD 58, RFC 2578, April 1999.

[RFC2579] MCCloghrie, K., Perkins, D., Schoenwaelder, J., Case, J., Rose, M. and S. Waldbusser, "Textual Conventions for SMIv2", STD 58, RFC 2579, April 1999.

[RFC2580] McCloghrie, K., Perkins, D., Schoenwaelder, J., Case, J., Rose, M. and S. Waldbusser, "Conformance Statements for SMIv2", STD 58, RFC 2580, April 1999.

[RFC2863] MCCloghrie, K. and F. Kastenholz, "The Interfaces Group MIB using SMIv2", RFC 2863, June 2000.

[IEEE802.3] IEEE, IEEE Std 802.3, 2002 Edition: "Carrier Sense Multiple Access with Collision Detection (CSMA/CD) Access Method and Physical Layer Specifications", March 2002 . 
[IEEE802.3ae] IEEE, IEEE Std 802.3ae-2002, "Amendment: Media Access Control (MAC) Parameters, Physical Layers, and Management Parameters for $10 \mathrm{~Gb} / \mathrm{s}$ Operation", August, 2002 .

[RFC3636] Flick, J., "Definitions of Managed Objects for IEEE 802.3 Medium Attachment Units (MAUs) using SMIv2", RFC 3636, September 2003.

8. Informative References

[RFC1212] Rose, M. and K. MCCloghrie, Editors, "Concise MIB Definitions", STD 16, RFC 1212, March 1991.

[RFC1213] MCCloghrie, K. and M. Rose, Editors, "Management Information Base for Network Management of TCP/IP-based internets: MIB-II", STD 17, RFC 1213, March 1991.

[RFC1284] Cook, J., "Definitions of Managed Objects for Ethernet-Like Interface Types", RFC 1284, December 1991 .

[RFC1369] Kastenholz, F., "Implementation Notes and Experience for The Internet Ethernet MIB", RFC 1369, October 1992.

[RFC1398] Kastenholz, F., "Definitions of Managed Objects for the Ethernet-like Interface Types", RFC 1398, January 1993.

[RFC1643] Kastenholz, F., "Definitions of Managed Objects for the Ethernet-like Interface Types", STD 50, RFC 1643, July 1994 .

[RFC1650] Kastenholz, F., "Definitions of Managed Objects for the Ethernet-like Interface Types using SMIv2", RFC 1650, August 1994 .

[RFC2358] Flick, J. and J. Johnson, "Definitions of Managed Objects for the Ethernet-like Interface Types", RFC 2358, June 1998 .

[RFC2665] Flick, J. and J. Johnson, "Definitions of Managed Objects for the Ethernet-like Interface Types", RFC 2665, August 1999.

[RFC2666] Flick, J., "Definitions of Object Identifiers for Identifying Ethernet Chip Sets", RFC 2666, August 1999. 
[RFC3410] Case, J., Mundy, R., Partain, D. and B. Stewart, "Introduction and Applicability Statements for Internet-Standard Network Management Framework", RFC 3410, December 2002 .

[CASE] Case, J., and C. Partridge, "Case Diagrams: A First Step to Diagrammed Management Information Bases", Computer Communications Review, 19(1):13-16, January 1989 .

[RFC3637] Heard, C., "Definitions of Managed Objects for the Ethernet WAN Interface Sublayer", RFC 3637, September 2003 .

[KASTEN] Kastenholz, F., "IEEE 802.3 Layer Management Draft compatible MIB for TCP/IP Networks", electronic mail message to mib-wg@nnsc.nsf.net, 9 June 1989.

9. Security Considerations

There is one management object defined in this MIB that has a MAXACCESS clause of read-write. That object, dot3PauseAdminMode, may be used to change the flow control configuration on a network interface, which may result in dropped packets, or sending flow control packets on links where the link partner will not understand them. Either action could be detrimental to network performance.

Such objects may be considered sensitive or vulnerable in some network environments. The support for SET operations in a non-secure environment without proper protection can have a negative effect on network operations.

Some of the readable objects in this MIB module (i.e., objects with a MAX-ACCESS other than not-accessible) may be considered sensitive or vulnerable in some network environments. In particular, the dot3statsEtherchipset object may be considered sensitive in many environments, since it would allow an intruder to obtain information about which vendor's equipment is in use on the network. Note that this object has been deprecated. However, some implementors may still choose to implement it for backwards compatability.

Most of the objects in this MIB module contain statistical information about particular network links. In some network environments, this information may be considered sensitive. 
It is thus important to control even GET and/or NOTIFY access to these objects and possibly to even encrypt the values of these objects when sending them over the network via SNMP.

SNMP versions prior to SNMPv3 did not include adequate security. Even if the network itself is secure (for example by using IPSec), even then, there is no control as to who on the secure network is allowed to access and GET/SET (read/change/create/delete) the objects in this MIB module.

It is recommended that the implementors consider the security features as provided by the SNMPv3 framework (see [RFC3410], section 8), including full support for the SNMPv3 cryptographic mechanisms (for authentication and privacy).

Furthermore, deployment of SNMP versions prior to SNMPv3 is NOT RECOMMENDED. Instead, it is RECOMMENDED to deploy SNMPv3 and to enable cryptographic security. It is then a customer/operator responsibility to ensure that the SNMP entity giving access to an instance of this MIB module is properly configured to give access to the objects only to those principals (users) that have legitimate rights to indeed GET or SET (change/create/delete) them.

\section{IANA Considerations}

This document does not define any new name space to be administered by IANA. However, section 3.2.4 does specify that some of the defined values in a current IANA-maintained name space are to be marked as deprecated or obsolete. In particular, the following enumerated values in the IANAifType TEXTUAL-CONVENTION in the IANAifType-MIB module have had an ASN.1 comment added by IANA stating that they have been deprecated:

- iso88032Csmacd(7)

- starLan (11)

In addition, the following enumerated values have had an ASN.1 comment added by IANA stating that they are obsolete:

- fastEther(62)

- fastEtherFX(69)

- gigabitEthernet (117)

In all of the above cases, the ASN.1 comment indicates that ethernetCsmacd(6) should be used instead of these values. 


\section{A. Change Log}

\section{A.1. Changes since RFC 2665}

This section enumerates changes made to RFC 2665 to produce this document.

(1) Updated references to the IEEE 802.3 standard to refer to the 2002 edition.

(2) Added reference to IEEE 802.3ae-2002.

(3) Updated WG e-mail address.

(4) The following DESCRIPTION clauses have been updated to reflect behaviour on $10 \mathrm{~Gb} / \mathrm{s}$ interfaces: dot3StatsAlignmentErrors and dot3statsSymbolErrors.

(5) The following objects have been added for management of the Rate Control function in WAN applications of ethernet: dot3statsRateControlAbility and dot3statsRateControlstatus.

(6) The following 64-bit counters have been added to support operation on high-speed ethernet interfaces: dot 3 HCControl InUnknownopcodes, dot3HCInPauseFrames, dot3HCOutPauseFrames, dot3HCStatsAlignmentErrors, dot3HCStatsFCSErrors, dot3HCStatsFrameTooLongs, dot3HCStatsInternalMacTransmitErrors, dot3HCStatsInternalMacReceiveErrors, dot 3 HCStats Symbolerrors

(7) Object groups and compliances have been added to contain the new objects.

(8) The MODULE-IDENTITY clause has been updated to reflect the changes in the MIB module.

(9) Use of the various iftype values for ethernet has been clarified to emphasize that all ethernet-like interfaces must use the ethernetcsmacd iftype.

(10) Several clarifications were made to the section on the mapping of the Interface MIB objects to ethernet.

(11) MIB boilerplate in section 2 has been updated to the latest approved text. 
A.2. Changes between RFC 2358 and RFC 2665

This section enumerates changes made to RFC 2358 to produce RFC 2665.

(1) Section 2 has been replaced with the current SNMP Management Framework boilerplate.

(2) The ifMtu mapping has been clarified.

(3) The relationship between the IEEE 802.3 octet counters and the IF-MIB octet counters has been clarified.

(4) REFERENCE clauses have been updated to reflect the actual IEEE 802.3 managed object that each MIB object is based on.

(5) The following object DESCRIPTION clauses have been updated to reflect that they do not increment in full-duplex mode: dot3statsSinglecollisionframes, dot3statsMultipleCollisionframes, dot3statsSQETestErrors, dot3statsDeferredTransmissions, dot3statslateCollisions, dot3statsExcessiveCollisions, dot3statsCarrierSenseErrors, dot 3 Collfrequencies.

(6) The following object DESCRIPTION clauses have been updated to reflect behaviour on full-duplex and $1000 \mathrm{Mb} / \mathrm{s}$ interfaces: dot3statsAlignmentErrors, dot3statsFCSErrors, dot3StatsSQETestErrors, dot3statslateCollisions, dot3statssymbolErrors.

(7) Two new tables, dot3ControlTable and dot3PauseTable, have been added.

(8) A new object, dot3statsDuplexstatus, has been added.

(9) The object groups and compliances have been restructured.

(10) The dot3StatsEtherChipset object has been deprecated.

(11) The dot3chipsets have been moved to a separate document.

A.3. Changes between RFC 1650 and RFC 2358

This section enumerates changes made to RFC 1650 to produce RFC 2358 .

(1) The MODULE-IDENTITY has been updated to reflect the changes in the MIB. 
(2) A new object, dot3statsSymbolerrors, has been added.

(3) The definition of the object dot3statsIndex has been converted to use the SMIV2 OBJECT-TYPE macro.

(4) A new conformance group, etherStats100MbsGroup, has been added.

(5) A new compliance statement, etherlo0Mbscompliance, has been added.

(6) The Acknowledgements were extended to provide a more complete history of the origin of this document.

(7) The discussion of iftype has been expanded.

(8) A section on mapping of Interfaces MIB objects has been added.

(9) A section defining the relationship of this MIB to the MAU MIB has been added.

(10) A section on the mapping of IEEE 802.3 managed objects to this MIB and the Interfaces MIB has been added.

(11) Converted the dot3Tests, dot3Errors, and dot3chipsets OIDs to use the OBJECT-IDENTITY macro.

(12) Added to the list of registered dot3chipsets.

(13) An intellectual property notice and copyright notice were added, as required by RFC 2026 .

Author's Address

John Flick

Hewlett-Packard Company

8000 Foothills Blvd. M/S 5557

Roseville, CA 95747-5557

Phone: +19167854018

EMail: johnferose.hp.com 
Full Copyright statement

Copyright (C) The Internet Society (2003). All Rights Reserved.

This document and translations of it may be copied and furnished to others, and derivative works that comment on or otherwise explain it or assist in its implementation may be prepared, copied, published and distributed, in whole or in part, without restriction of any kind, provided that the above copyright notice and this paragraph are included on all such copies and derivative works. However, this document itself may not be modified in any way, such as by removing the copyright notice or references to the Internet society or other Internet organizations, except as needed for the purpose of developing Internet standards in which case the procedures for copyrights defined in the Internet Standards process must be followed, or as required to translate it into languages other than English.

The limited permissions granted above are perpetual and will not be revoked by the Internet society or its successors or assignees.

This document and the information contained herein is provided on an "AS IS" basis and THE INTERNET SOCIETY AND THE INTERNET ENGINEERING TASK FORCE DISCLAIMS ALL WARRANTIES, EXPRESS OR IMPLIED, INCLUDING BUT NOT LIMITED TO ANY WARRANTY THAT THE USE OF THE INFORMATION HEREIN WILL NOT INFRINGE ANY RIGHTS OR ANY IMPLIED WARRANTIES OF MERCHANTABILITY OR FITNESS FOR A PARTICULAR PURPOSE.

Acknowledgement

Funding for the RFC Editor function is currently provided by the Internet society. 